\title{
Twenty-first century brain banking. Processing brains for research: the Columbia University methods
}

\author{
Jean Paul G. Vonsattel - Maria Pilar del Amaya • \\ Christian E. Keller
}

Received: 8 August 2007/Revised: 11 October 2007 / Accepted: 14 October 2007/Published online: 6 November 2007

(c) Springer-Verlag 2007

\begin{abstract}
Carefully categorized postmortem human brains are crucial for research. The lack of generally accepted methods for processing human postmortem brains for research persists. Thus, brain banking is essential; however, it cannot be achieved at the cost of the teaching mission of the academic institution by routing brains away from residency programs, particularly when the autopsy rate is steadily decreasing. A consensus must be reached whereby a brain can be utilizable for diagnosis, research, and teaching. The best diagnostic categorization possible must be secured and the yield of samples for basic investigation maximized. This report focuses on integrated, novel methods currently applied at the New York Brain Bank, Columbia University, New York, which are designed to reach accurate neuropathological diagnosis, optimize the yield of samples, and process fresh-frozen samples suitable for a wide range of modern investigations. The brains donated for research are processed as soon as possible after death. The prosector must have a good command of the neuroanatomy, neuropathology, and the protocol. One half of each brain is immersed in formalin for performing the thorough neuropathologic evaluation, which is combined with the teaching task. The contralateral
\end{abstract}

\footnotetext{
J. P. G. Vonsattel · M. P. del Amaya - C. E. Keller

The Taub Institute for Research on Alzheimer's Disease and the Aging Brain, The Department of Pathology in the College of Physicians and Surgeons, Columbia University, New York, USA
}

\section{J. P. G. Vonsattel $(\bowtie)$}

The New York Brain Bank,

Columbia University Children's Hospital,

Tunnel T8, 3959 Broadway, New York, NY 10032, USA

e-mail: jgv2001@ columbia.edu half is extensively dissected at the fresh state. The anatomical origin of each sample is recorded using the map of Brodmann for the cortical samples. The samples are frozen at $-160^{\circ} \mathrm{C}$, barcode labeled, and ready for immediate disbursement once categorized diagnostically. A rigorous organization of freezer space, coupled to an electronic tracking system with its attached software, fosters efficient access for retrieval within minutes of any specific frozen samples in storage. This report describes how this achievement is feasible with emphasis on the actual processing of brains donated for research.

\section{Introduction}

The psychological and financial burdens of neurodegenerative diseases increasingly strain the familial and social framework of our societies. This trend is linked to the gradual lengthening of life expectancy and is therefore worsening [25]. Thus, ongoing efforts to lessen the burden of neurodegeneration must be improved both qualitatively and quantitatively. Many neurodegenerative diseases occur exclusively in humans. For this reason, the natural course of their pathogenesis can only be methodically and thoroughly investigated within the human brain. Therefore, the availability of carefully categorized postmortem human brains is crucial for stripping bare the complex cascade of the deleterious mechanisms leading, for example, to dementia or movement disorders. Thus, it is essential to promote brain donation for research [13, 45, 51].

The lack of generally accepted methods for processing human postmortem brains for research persists despite the huge advancements in brain banking $[4,7,16-18,27,28$, 30, 37, 40, 43, 47, 49]. Thus, developing standardized 
methods and networking existing brain banks of academic centers are the prerequisites to meet the growing requirements of modern research. This constellation of wellcoordinated efforts of brain donation, processing, storage, and research is crucial for alleviating the miseries of neurodegeneration.

Nevertheless, brain banking cannot be achieved at the cost of the teaching mission of academic institutions by routing brains away from residency programs, particularly when the autopsy rate is steadily decreasing [24, 39]. A compromise must be worked out whereby a brain can be utilizable for diagnosis, research, and teaching.

Above all, any human brain donated for research must be accurately categorized according to clinical and pathological data. The best diagnostic categorization possible must be secured and reached, at the same time maximizing the yield of samples for basic investigations. Thus, carrying out reliable, reproducible methods is all-important.

Electronically recording either the absence of changes or the type, distribution, and extent of changes in brains banked, is imperative to best meet the precise requirements of explicit investigations in a qualitative, timely manner. Electronically tracking the samples to monitor their destiny greatly enhances the capability of brain banking. Indeed, coupling the electronic tracking system with a database including the diagnostic characteristics of the stored blocks assists in sorting out a set of samples that best match the parameters of a tissue request. Additionally, electronic tracking of the methodically categorized samples is essential for swift retrieval of those that are suitable for a specific request among the ones kept in freezers.

The methods currently applied at the New York Brain Bank-Columbia University, New York-are meeting these challenges. They were developed along basic guidelines provided by pioneers of brain banking, including, among others, Drs. E. D. Bird, N. J. Cairns, F. F. CruzSanchez, R. Faull, M. Graeber, C. M. Hulette, K. Jellinger, R. Ravid, and W. T. Tourtellotte. The late Dr. E. P. Richardson and Dr. E. T. Hedley-Whyte were central in combining the tasks of postmortem neuropathologic evaluation, teaching, and brain banking.

The methods reported here have been gradually constructed, improved, and implemented since 2001, thanks to strong support from Columbia University, the Taub Institute under the leadership of Dr. Michael Shelanski and Dr. Richard Mayeux, and the Hereditary Disease Foundation. These methods are adequate for achieving reliable diagnostic assessments, efficient teaching, and obtaining many well-characterized and optimally prepared brain samples that fulfill the requirements of modern research, especially on neurodegeneration.

A rigorous organization of freezer space, coupled to an electronic tracking system with its attached software, fosters efficient access for retrieval within minutes of any specific frozen sample among more than 150,000 currently in storage. In 2006, the monthly mean of samples disbursed to eligible neuroscientists worldwide was up to 500. The monthly mean of source brains for the corresponding disbursement of samples was 150 . The time line between the receipt of a request and the actual disbursement of samples was five working days. This report describes how this achievement is feasible with emphasis on the processing of brains donated for research.

Briefly, modern methods of brain banking must make the following crucial aims attainable:

- To accomplish a thorough postmortem examination for reaching the best neuropathological categorization

- To teach students and residents

- To harvest for research as many samples as possible from brains donated in such a way that each sample can be disbursed immediately, once categorized diagnostically

- To track the samples stored using a set of variables for sorting out those among $>100,000$ that best match the specific requirements of a wide range of investigations.

To achieve these aims, the brains donated for research must be optimally processed as soon as possible after death. The prosector must have a good command of the neuroanatomy, neuropathology, and the protocol. After harvesting the cerebrospinal fluid (CSF), one-half of each brain is immersed in formalin for performing thorough neuropathologic evaluation, which is combined with the teaching task. The contralateral half is extensively dissected in the fresh state. Up to 150 or more fresh samples are harvested per half-brain, either as blocks or as pulverized aliquots of parenchyma. The anatomical origin of each sample is recorded using the map of Brodmann for the cortical samples [11]. The samples are frozen using liquid nitrogen vapor (LNV) at $-160^{\circ} \mathrm{C}$, and barcode labeled. Currently, three measurable parameters serve to assess the quality of the tissue banked: the $\mathrm{pH}$ value, the yield of total RNA per unit of tissue, and the extent of degradation of ribosomal RNA.

Thus, samples banked are: (1) LNV fresh frozen blocks (size range between $0.3 \times 0.5 \times 1.0 \mathrm{~cm}$ and $0.5 \times 2.5 \times$ $3.0 \mathrm{~cm}$ ), which are especially suitable for studies requiring preservation of the cellular morphology and that of the cytoarchitecture of the area of interest; (2) aliquots of fresh-frozen pulverized brain parenchyma $(1.0-1.5 \mathrm{ml})$, especially for studies focusing on biochemistry, protein studies, or molecular biology; (3) fresh-frozen CSF aliquots (1.0-1.5 ml); (4) fresh-frozen miscellaneous tissues (e.g., pineal gland, pituitary gland, choroid plexus, vessels); (5) a standardized series of 18 buffered formalin 
phosphate-fixed and paraffin-embedded blocks, and spinal cord; and (6) $10 \%$ buffered formalin phosphate-fixed remnants of brain and spinal cord that are left from the dissection.

This report focuses on integrated methods (referred to as "Protocol 1") of processing brains donated for research, which are designed to: (1) reach accurate neuropathological diagnosis; (2) optimize the yield of samples; and (3) process fresh-frozen samples suitable for a wide range of modern investigations, using either traditional or the latest technologies. These methods are now applied successfully at the New York Brain Bank (NYBB) of Columbia University. Indeed, our novel methods optimize the yield of precious human brain tissue for research, secure the postmortem diagnostic procedure, and foster teaching opportunities for scientists in training, medical students, residents, fellows, and health professionals. Although the logistics of the electronic tracking system and combined software are essential operative assets of the NYBB, only key relevant points will be addressed in this report. Not addressed in this report are the safety precautions and the issues of confidentiality that must be applied in human tissue repository for research.

\section{The facilities and personnel}

The laboratories of the NYBB are next to the autopsy suite of the New York Presbyterian Hospital. The operation of the NYBB is fully integrated with the functions of the Department of Pathology and Neuropathology of the New York Presbyterian Hospital. This integration fosters the teaching tasks of the department, and prevents the duplication of efforts in the histology laboratory for processing the tissue used for microscopic evaluation of the banked brains. Thus, each brain donated to the NYBB is an opportunity to establish definitive postmortem diagnoses, teach trainees, and harvest samples for research.

The operational space assigned to the NYBB consists of five and one-half rooms: (1) storage freezer room $\left(32.0 \mathrm{~m}^{2}\right)$, (2) laboratory $\left(35.0 \mathrm{~m}^{2}\right)$, (3) dissection room $\left(16.6 \mathrm{~m}^{2}\right)$, (4) storage room for formalin-fixed samples $\left(3.9 \mathrm{~m}^{2}\right),(5)$ office of the director $\left(27.0 \mathrm{~m}^{2}\right)$, and (6) one shared room for administration $\left(8.0 \mathrm{~m}^{2}\right)$. The total surface of these rooms is $122.5 \mathrm{~m}^{2}$.

The personnel include a laboratory supervisor, an administrative assistant, a postdoctoral research scientist, and a board-certified neuropathologist, who is the director and who has as an appointment in the Department of Pathology. The director and the postdoctoral research scientist, both MDs, perform the dissection of the brains and are on call $24 \mathrm{~h} / 7$ days.

\section{Equipment used for Protocol 1}

To prepare a brain according to Protocol 1 , we use the following items:

1. A double-walled vacuum vessel (XLC140);

2. One pair of Teflon-coated aluminum plates $(1.0 \times 8.0 \times 10.0 \mathrm{~cm})$;

3. A cold surface to lay the freshly sectioned slices;

4. A porcelain mortar (Fisher Catalog 60319) with a pestle (Fisher Catalog 60320);

5. A spherical Dewar flask (Fischer Catalog 10-196-C, code 5694);

6. "Polyfoam Insulated Storage Chest" with dry ice;

7. Plastic bags "Bitran SX $2 \times 4$, reclosable, leakproof" (Fisher Catalog 19240093; MFG\#: FSB4740);

8. Polypropylene "Wheaton Cryule, $2.0 \mathrm{ml}$ " vials (Fisher catalog 03-341-18H, E, G);

9. Boxes (Fisher catalogue 5954) to keep either frozen blocks in plastic bags (no. 7 above), or brain aliquots in vials (no. 8 above);

10. $5.0 \mathrm{ml}$ Syringe "Luer-Lok" (Fisher catalog 309603).

Accessories for slicing the brainstem and cerebral hemisphere:

11. Slide holder wrapped with gauze;

12. Cutting ice support (CIS) with two aluminum plates, one facing the cut surfaces, and one for abutment;

13. Brain cutting board; 1 knife (Victorinox, 455-14,R.H. Forschner Co.); scalpels, safety razor blades, spatula, and photographic setup.

Details on specific items used will be provided with the proceedings implicating them.

\section{Upon reception of the fresh brain}

Only professionals who are familiar with neuroanatomy, neuropathology, and have a good command of the protocol perform the dissection of the brain to minimize the probability of overlooking unexpected changes [31]. Usually, two individuals are involved with the dissection, which lasts between 60 and $90 \mathrm{~min}$.

The whole fresh brain is examined, photographed, and weighed. Any identified abnormality is recorded. Then the CSF is obtained. To harvest the CSF, the whole, fresh brain is placed on the cutting board with the ventral aspect facing the prosector and with the frontal poles away from the prosector. A transverse cut is performed through the distal end of the infundibulum. Then, the distally truncated safety needle cap attached to a clean $5 \mathrm{ml}$ syringe is inserted into 
the infundibulum. The CSF is drawn up into the syringe. To increase the yield of CSF, the occipital lobes are gently raised to drain the CSF toward the infundibulum. Then, aliquots of the CSF are generated using an appropriate number of barcoded vials, each containing about $1.5 \mathrm{ml}$. The olfactory bulb(s), pineal gland, and optic nerve are then harvested, frozen, and placed in separate, barcoded vials.

\section{Dividing the brain}

Two variants are used: (1) usual (Fig. 1), that includes two options; and (2) amyotrophic lateral sclerosis variant (Fig. 2). For the usual variant, a sagittal cut is performed either in a dorsoventral direction through the corpus callosum first, or in a ventrodorsal direction, first through the midline of the brainstem and then through the corpus callosum.
The sectioning in a ventrodorsal direction is recommended to secure a perfect sagittal split of the brain. If the section is performed in a dorsoventral direction, the following precautions should be taken (Fig. 1a). Before sectioning, one should make sure that: (1) a gap exists between the frontal lobes; (2) the medulla oblongata is aligned so that it is in perfect continuity with the rostrocaudal axis of the corpus callosum; (3) the cut must be perpendicular to the cutting board; (4) the knife is pulled smoothly, possibly with one stroke, using the full length of the blade. A slightly oblique sagittal cut will result in having the hypothalamus, raphe nucleus, or the 12th cranial nerve nucleus absent or incomplete in one half-brain. This problem can be avoided if the brain is sectioned in a ventrodorsal direction after placing rolled gauze above the corpus callosum to keep the hemispheres apart (Fig. 1b-d).

To unequivocally obtain the 12th cranial nerve nuclei bilaterally in alternate blocks that are either frozen and banked, or fixed for microscopic evaluation, a modification
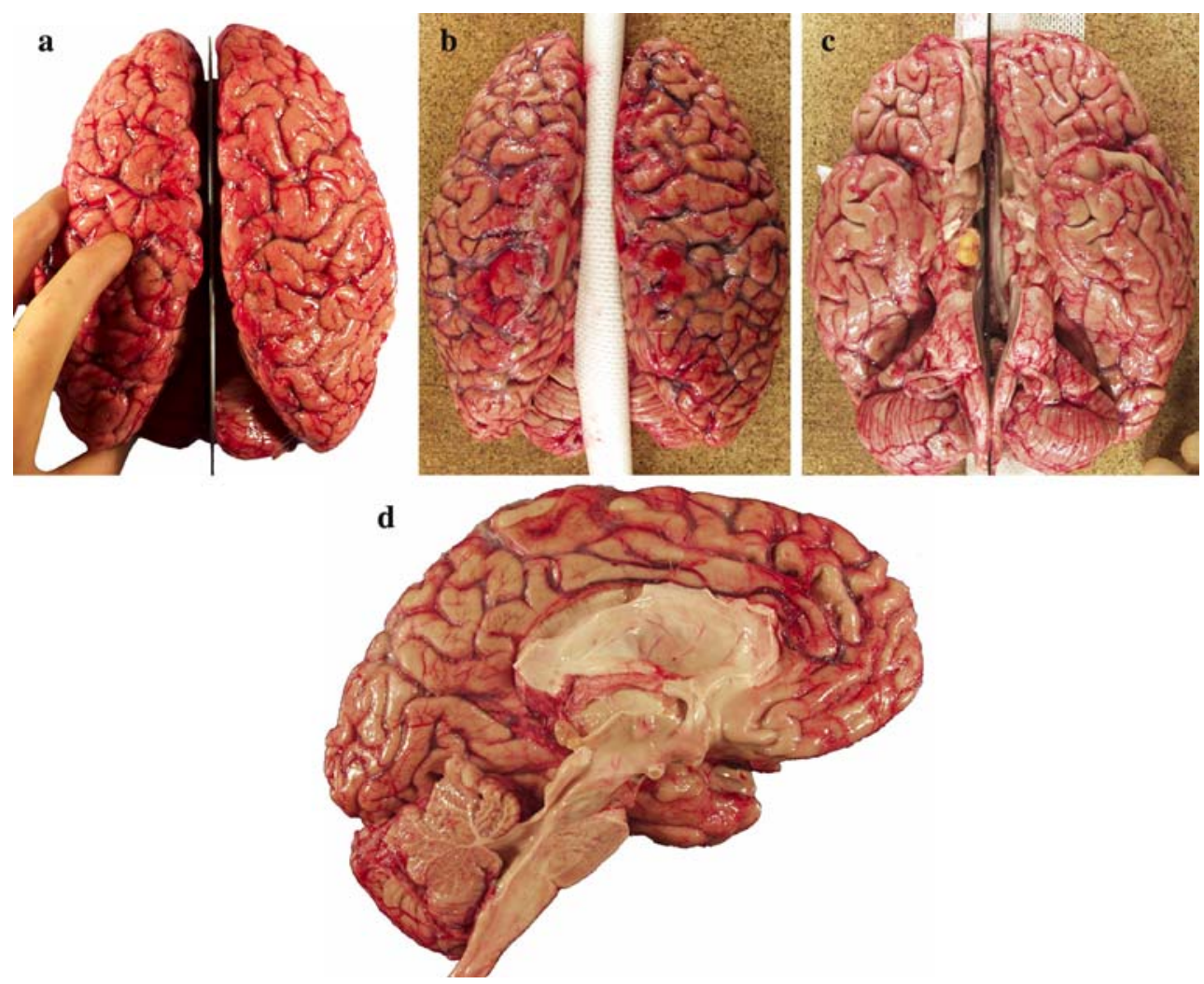

Fig. 1 Usual variant for sectioning the brain sagittally. The dorsoventral sagittal cut through the corpus callosum is the simplest variant; however, it requires experience to obtain a cut passing exactly through the midline (a). To secure a section passing perfectly through the midline, four sheets of gauze are rolled together along their longitudinal axis, stretched, and carefully apposed to the dorsal aspect of the corpus callosum (b). The brain is rotated so that its dorsal aspect is resting on the cutting board. Then the knife is carefully placed along the midline of the brainstem and between the frontal lobes, ventrally, and then the cut proceeds, possibly with one stroke, using the full length of the blade. Note the presence of a lipoma attached to the right, lateral aspect of the infundibulum (c). Medial aspect of the left halfbrain sectioned in a ventrodorsal direction after having placed the gauze belt over the corpus callosum (d) 

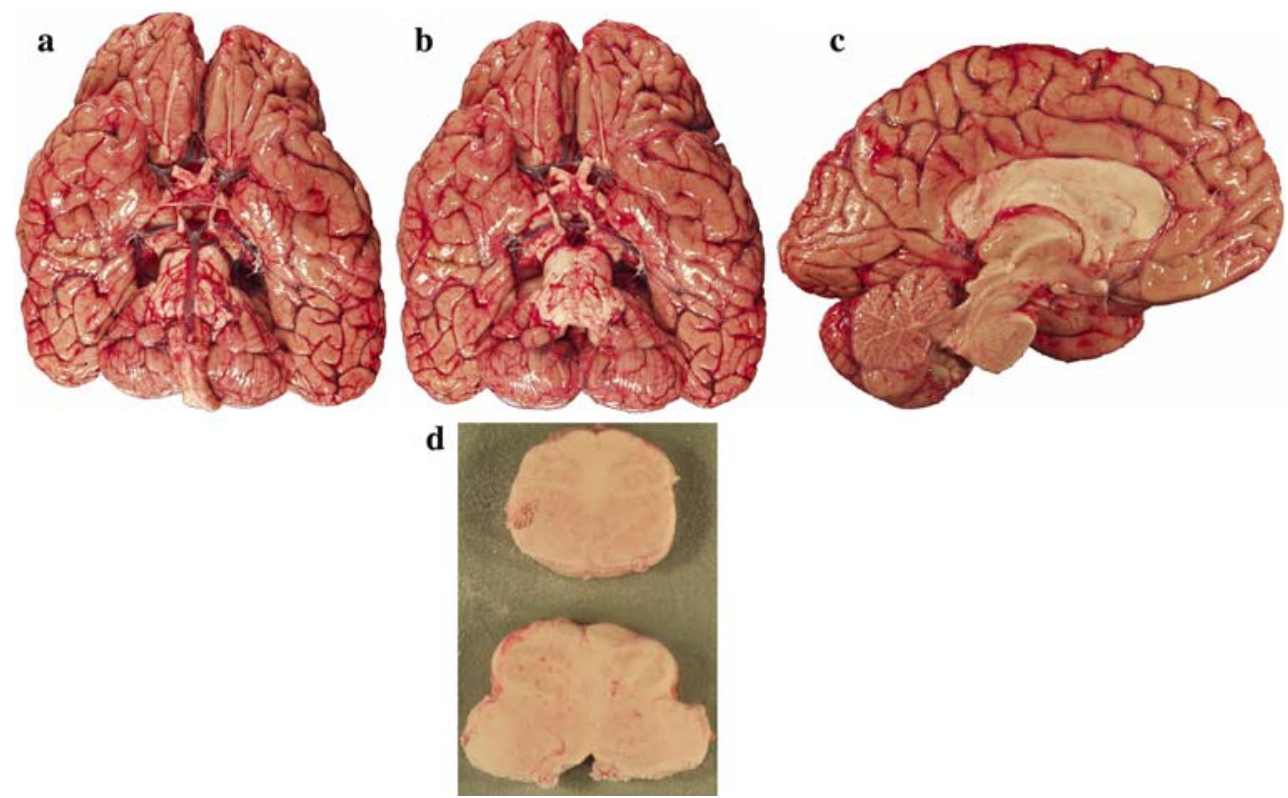

Fig. 2 Variant applied when processing brains of patients with amyotrophic lateral sclerosis, which is referred to as "ALS variant of Protocol 1" (P1-ALS variant). This variant secures the presence of the bilateral nucleus of the hypoglossal nerve (XII) in blocks used either for research (frozen) or diagnostic (formalin fixed). The

of the protocol is applied, which is referred to as "P1-ALSvariant." This variant is applied when processing brains of patients with amyotrophic lateral sclerosis (ALS). At the fresh state, before performing the sagittal split of the brain through the corpus callosum, the myelencephalon is separated transversally from the metencephalon (Fig. 2a-c). Then, four, $0.3 \mathrm{~cm}$-thick transverse slices of the myelencephalon are obtained; two of them are formalin fixed for the neuropathologic evaluation, and two are frozen and banked (Fig. 2d). Details on how to obtain these blocks are provided below.

\section{Laterality}

The selection of which half-brain is prepared for research depends on the numerical day on which the fresh brain is received for processing (e.g., even day, right half; odd day, left half). However, if a unilateral lesion is detected on gross examination of the fresh brain, the side involved is formalin fixed. In instances in which the extent of the degenerative changes is asymmetric, as in corticobasal degeneration (CBD) for example, the more severely atrophic side is kept for neuropathological evaluation.

In the absence of a focal lesion or symptoms indicative of a localized, concomitant pathologic process, it is assumed that the findings in one half-brain reflect the parenchymal state of the contralateral half-brain, which is myelencephalon (a) is detached from the metencephalon (b) before the sagittal cut through the corpus callosum $(\mathbf{c})$. Then $0.3 \mathrm{~cm}$-thick transverse slices are obtained from the myelencephalon. Alternate slices are frozen $(\mathbf{d})$ or fixed (not shown)

verified while processing the fixed counterpart. If the cut surface of any freshly processed slice shows unexpected changes, that sample is immersed in formalin. Thus, it is important that a trained professional processes the fresh half-brain at this seminal stage of brain banking. One must guarantee that the samples made available to neuroscientists harbor the anomalies proper to the disease investigated, or are eligible as control.



Fig. 3 One half-brain is processed fresh. Up to 150 blocks and parenchymal aliquots are harvested and barcode labeled (de-identified link and site of origin). The contralateral half is fixed for neuropathological evaluations 

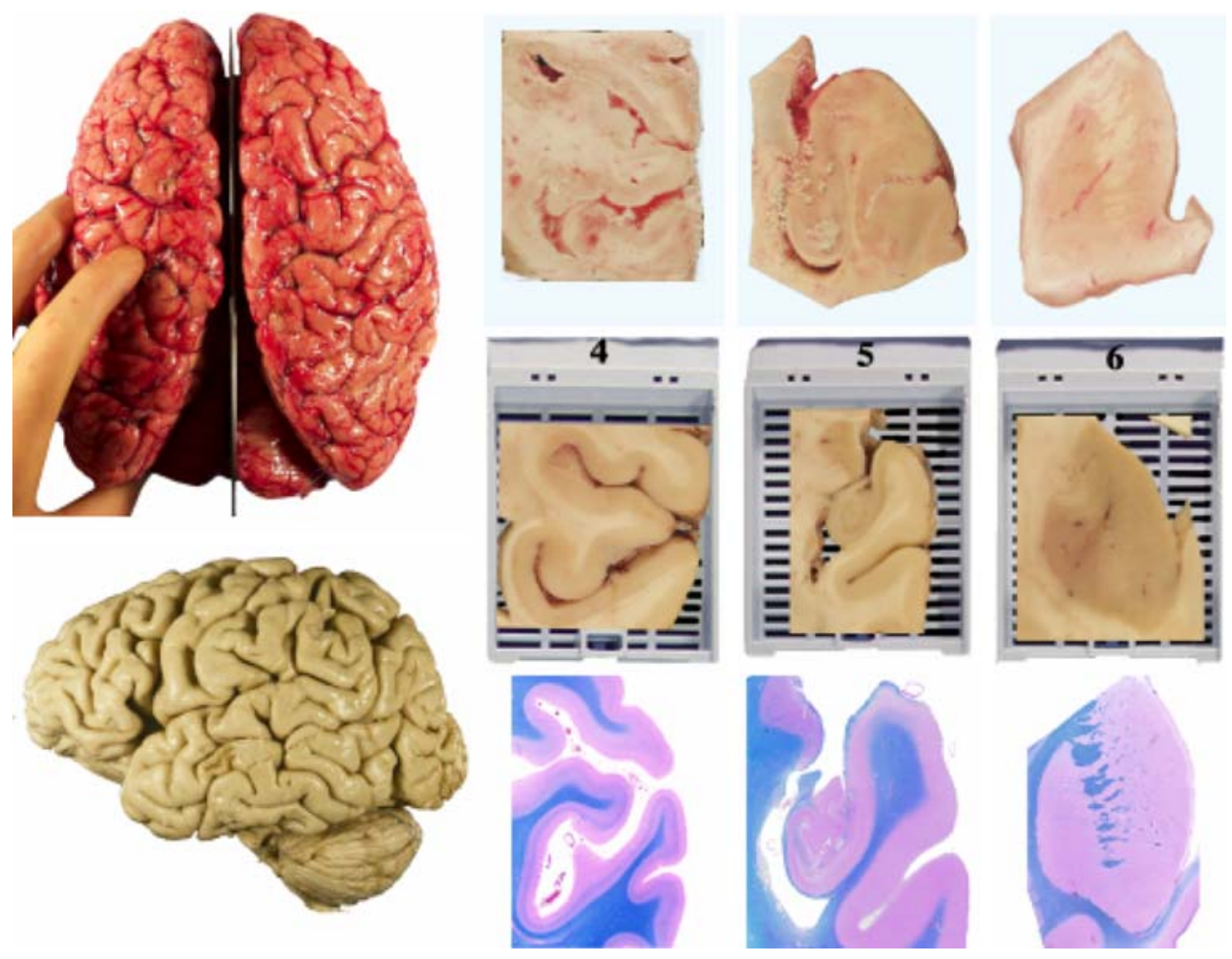

Fig. 4 Standard brain blocks (SBB): a series of 36 blocks are obtained from 18 precisely selected areas of each half-brain (see Figs. 12, 22). Representative, frozen blocks (SBB4, 5, 6), obtained from the fresh half-brain (upper right) or from the fixed half-brain (middle right), from which Luxol fast blue counterstained with hematoxylin and eosin $7 \mu \mathrm{m}$ thick sections are produced for microscopic evaluation (lower right), are perfectly matched. See text
Figure 3 summarizes the fate of the half brain prepared for research, and that of the contralateral half used to reach the best diagnostic categorization, as an integrated part of the teaching mission of an academic center.

Multiple sampling eases the reproducibility of experiments using blocks of tissue from the same brain by the same team, or among diverse teams of investigators, which is compellingly essential for validating results. Moreover, multiple sampling allows comparisons of data, which may derive from different Brodmann areas. This is a must for correlating findings obtained with functional neuroimaging methods, including tensor- or voxel-based morphometry. Multiple sampling expedites the disbursements of brain blocks or aliquots with accurate topographic and diagnostic distinctiveness.

Three series of blocks are harvested: (1) a series of 36 blocks obtained from 18 precisely selected areas of each half-brain, which is referred to as "standard brain blocks" (SBB). The 18 blocks to be frozen, which are obtained from the fresh half-brain (Fig. 4: representative fresh frozen blocks, top row, right), exactly match the complementary set of 18 blocks obtained from the fixed half-brain (Fig. 4: representative matching formalin fixed blocks, middle row, right), which is processed for microscopic examination
(Fig. 4: bottom row, right), and obtained from the fresh half-brain only; (2) a series of blocks, which includes the remainder of the same areas (homotopic extra series) as the SBB series defined above, without, however, matched counterparts from the fixed half-brain (e.g., additional levels including the visual cortex); and (3) a series of blocks (heterotopic extra series) referred to as "additional brain blocks" (ABB) [e.g., a block including Brodmann areas (BA) 11, 47] obtained outside the areas dealt with above. In addition to these three series of blocks, pulverized brain aliquots, pulverized in liquid nitrogen, are harvested and stored frozen in vials (see below).

\section{Sectioning the fresh half-brain}

The anatomical origin (e.g., Brodmann area) of each cortical sample that is banked is one of the factors used to identify any specimens in storage [11]. Thus, the dissection of the fresh brain must be meticulous and anatomically as precise as possible. Therefore, a few technical details are provided. Because the demand for the mesencephalon is far greater than its availability, two levels are obtained (Fig. 5). 


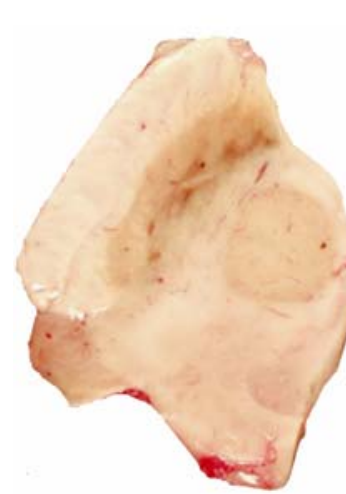

Fig. 5 Alternate, fresh, and LNV-frozen hemi mesencephalon: left, rostral fresh/frozen slices; right, caudal transverse fresh/frozen slices, each about $0.3 \mathrm{~cm}$ thick. The frozen slices are resting on a cold Teflon-coated aluminum plate (see text). The rostral level is

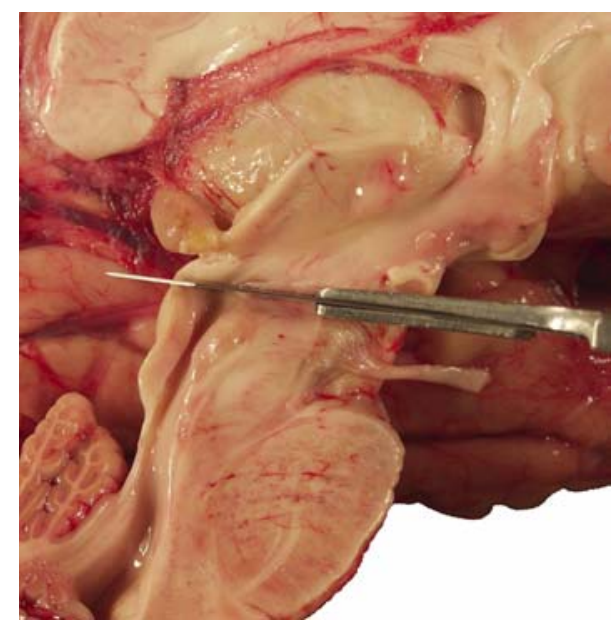

Fig. 6 Separation of the hemi brainstem with attached cerebellum from the cerebral hemisphere. The plan of the cut grazes the ventral edge of the mammillary body, and the dorsal edge of the superior colliculus

The rostral level includes the red nucleus with the pars reticulata $>$ pars compacta. The caudal level includes the decussation of the superior cerebellar peduncle with the pars compacta $>$ pars reticulata. To best obtain these two levels, the following technique (Amaya's version) is applied (Fig. 6): the hemi brainstem and hemi cerebellum from the fresh half-brain are detached from the cerebral hemisphere by a transverse cut through the rostral tip of the mesencephalon along a straight line, tangent to the ventral aspect of the mammillary body, and to the dorsal aspect of the superior colliculus (this line is parallel to the line passing between the superior and inferior colliculi, and the dorsal edge of the 3rd cranial nerve). Next, the hemi cerebellum is detached from the hemi brainstem by especially suitable for research focusing on progressive supranuclear palsy (PSP), and the caudal level for studies on Parkinson's disease (PD)

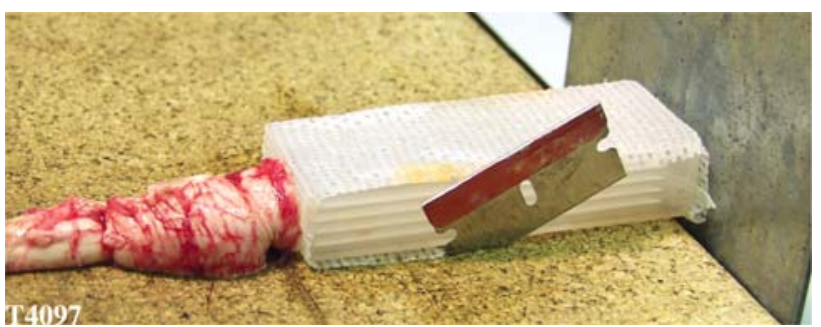

Fig. 7 Slide holder with gauze and hemi brainstem, the cut surface of which is applied against the bottom or lid of the stabilized slide holder to obtain regular, transverse, $0.3 \mathrm{~cm}$ thick slices. The razor blade and the vertical aluminum plate keep the slide holder steady

sequentially sectioning the superior, middle, and inferior cerebellar peduncles.

\section{Fresh hemi brainstem}

The best transverse slices of the hemi brainstem can be attained using a slide holder wrapped with a $3.0 \times 20.0 \mathrm{~cm}$ piece of gauze (Fig. 7), which has been moistened with $100 \%$ ethanol. Once carefully applied, the gauze is kept steady around the slide holder by locking the two ends while closing the lid. Then, the successive cut surfaces of the hemi brainstem are apposed against the bottom of the stabilized slide holder to sequentially obtain $0.3 \mathrm{~cm}$-thick transverse slices.

The cuts are performed gently with one stroke of the knife. The weight of the knife provides the vertical force for sectioning. Each cut is made within one stroke and is perpendicular to the cutting board, and parallel to the apposing surface of the slide holder. While cutting, the prosector, using his thumb and index finger in an upsidedown "U" shaped position, holds the anterior and posterior 
aspects of the brainstem as close as possible to the blade of the knife.

Fresh hemi cerebellum

The vermis is gently applied to the clean cutting board, and then sequential, $0.3 \mathrm{~cm}$-thick, sagittal slices are obtained. Two blocks, including, in a dorsoventral direction, the cortex, album cerebelli, and dentate nucleus, are banked as standard blocks. Additional blocks are taken as DNA reservoir, and aliquots of pulverized parenchyma, mainly the cortex.

\section{Fresh cerebral hemisphere}

To obtain optimal $0.5 \mathrm{~cm}$ thick coronal slices of the cerebral hemisphere, a carpeted "cutting ice support" (CIS) is used, against which the successive cut surfaces of the
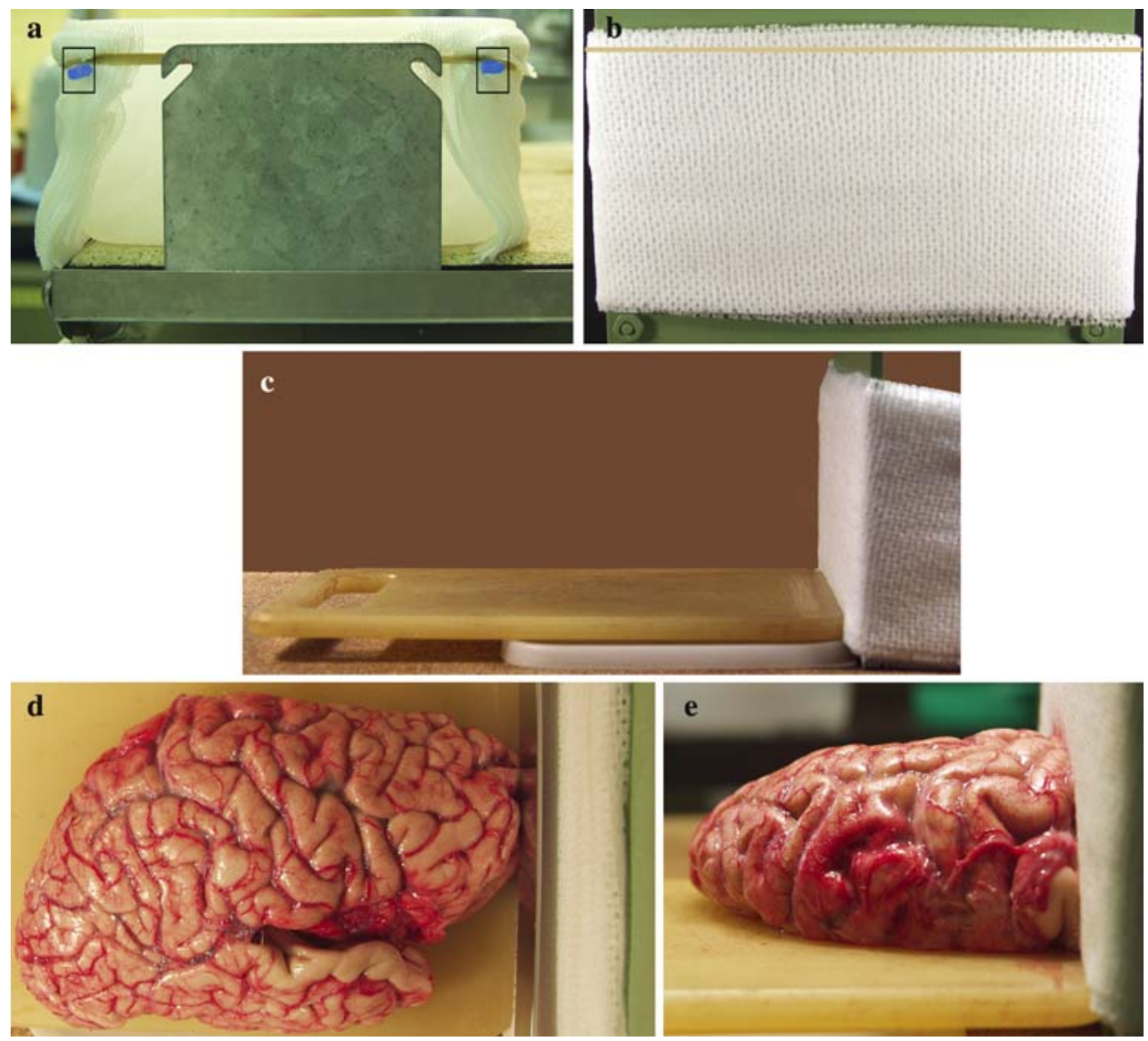

Fig. 8 The posterior aspect of the cutting ice support (CIS) includes two hooks (framed) to which the carpet of gauze is anchored; the vertical plate on the back stabilizes the CIS (a). Anterior aspect of the CIS (b): first, an aluminum plate is apposed vertically against the front of the ice-filled container to provide a perfect flat surface. Then, one end of the carpet of gauze is anchored to one hook, which is on the posterior aspect of the CIS (a). The carpet is stretched over the aluminum plate on the front, and anchored to the other hook of the CIS on the back. Thus, the stretched carpet evenly covers the aluminum plate apposed against the anterior aspect of the ice-filled container. A rubber band braces the carpet to steady it. Lateral view of the CIS (c). The CIS is resting on a cork cutting board and is apposed against the vertical aluminum plate, which is on the back (a). The cerebral hemisphere is placed on the movable plastic cutting board that abuts the front of the CIS and is slightly elevated to take full advantage of the vertical, carpeted surface (c). The long axis of the movable cutting board and the tangent of the ventral aspect of both the temporal and occipital lobes are aligned and strictly perpendicular to the anterior aspect of the CIS. These axes, the vertical carpeted surface of the CIS, and the long axis of the cork cutting board serve as guides while sectioning the cerebral hemisphere. The tangent to the ventral aspect of the temporal and occipital lobes of a right cerebral hemisphere is aligned with the long edge of the movable cutting board, and is near the right-handed prosector; the blade of the knife is parallel to the carpeted surface (d); conversely, the tangent is away from the right-handed prosector when a left cerebral hemisphere is processed (e). The long edges of the movable cutting board are kept perpendicular to the CIS and the cut surface of the hemisphere is evenly applied against the carpet (e) 
cerebral hemisphere will be apposed (Fig. 8). The CIS is kept frozen at $-5^{\circ} \mathrm{C}$, and is retrieved shortly before processing a brain.

The CIS consists of a modified plastic container $(18.0 \times 13.0 \times 8.0 \mathrm{~cm}$; Republic Molding Corp., Chicago, IL 60648, no. 334/3334). Two plastic hooks are placed, as shown in Fig. 8a. Holes are drilled; distal segments of safety needle caps (of $5.0 \mathrm{ml}$ syringe) are inserted within the holes in an inside-out direction. Once the hooks are positioned, the container is filled with water, and the lid tightly placed.

To achieve a perfectly even, vertical, cold area against which the cut surfaces of the cerebral hemisphere will be applied to obtain optimal slices, a $17.0 \times 14.0 \times 0.5 \mathrm{~cm}$ aluminum plate is placed between the front aspect of the ice-filled container and a carpet of gauze (Fig. 8b, c). This carpet consists of 10-15, or more layers of gauze that are carefully superposed, and moistened with $100 \%$ ethanol. Two "buttonholes" are made to fix to the hooks (Fig. 8a) and stretch the carpet. First, the frontal pole, and then the successive cut surfaces of the cerebral hemisphere, will be apposed against this stretched, flattened, cold carpet for the serial slicing (Fig. 8d, e).

Optimizing the yield of fresh frozen samples that are well characterized anatomically and diagnostically, and ready for immediate disbursement once categorized, is the quintessence of this protocol. The banked fresh frozen samples consist of blocks or aliquots of parenchyma obtained from precise anatomical areas, as defined by Brodmann for the cerebral cortex (Figs. 9, 10) [11]. As regards the cerebral hemisphere, this is achieved by obtaining a series of coronal slices, the ideal thickness of which is $0.5 \mathrm{~cm}$.

The extent of the harvest of fresh frozen samples depends on the impression of the quality of the brain (as can be assessed at the time of processing, e.g., consistency,

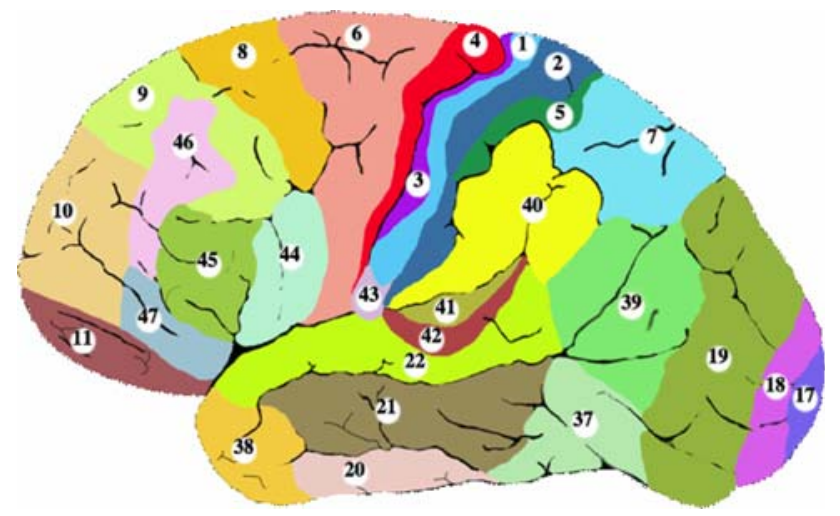

Fig. 9 The Brodmann areas (BA) are shown color-coded on the lateral aspect of the left cerebral hemisphere

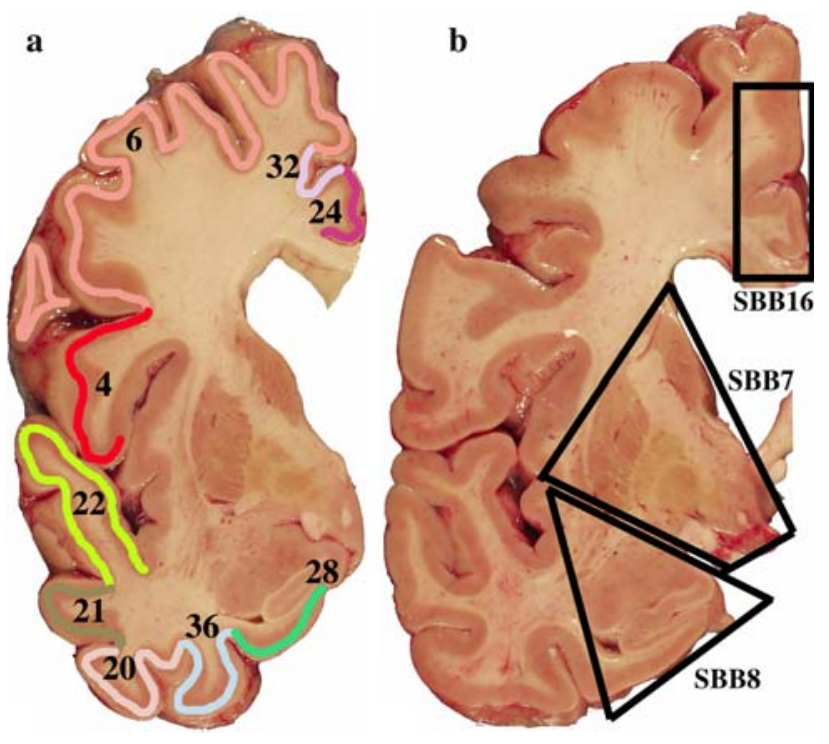

Fig. 10 Posterior aspects of fresh, coronal slices of two distinct hemispheres passing through the amygdala, as they appear on the cold plate (Fig. 11), ready for the harvest of either the standardized (SBB) or additional blocks (ABB), and of the aliquots (vials). The Brodmann areas (BA) are shown color-coded (a) and are used as one of the identifiers of the blocks that include the cortex. The framed areas represent the standardized brain blocks $S B B 8$ (amygdala), SBB7 (GP, globus pallidus), and SBB16 (cingulate gyrus) (b)

presence or absence of streaks of parenchyma on the blade of the knife), on the information on the premortem state of the patient, and on the inventory of the samples available in storage freezers. The half-brain of a clinically well-categorized individual, which is without evidence of autolysis, will be extensively dissected. Up to 150 or more samples will be obtained. In this instance, the deep gray nuclei and hemi-brainstem are entirely banked, the exception being minute portions that are trimmed from selected blocks so that their surfaces match that of a histology slide. If evidence of autolysis are noticed at the time of processing, a minimum of 66 fresh frozen samples are banked, which consist of blocks and pulverized parenchyma (vials). The advantage of this relative, selective practice is that frozen samples are stored from each brain donated. Furthermore, it allows prioritization of cabinet space of freezers, which is limited, according to the quality of the samples harvested.

\section{Positioning the cerebral hemisphere}

The cerebral hemisphere is cut coronally along an axis perpendicular to a line that is tangential to both the ventral aspect of the inferior temporal gyrus and the occipital pole (Fig. 8d). Either the medial or the lateral aspect of the hemisphere rests on the movable plastic cutting board in obtaining coronal slices. The stability of the cerebral 
hemisphere is optimal when its medial aspect, which is flat, rests on the cutting board. This option is best for obtaining $0.5 \mathrm{~cm}$-thick slices that are free of distortion. However, the discrete anatomical landmarks of the medial aspect of the hemisphere are not visible. Nonetheless, topographic accuracy of the standard blocks is secured because of the distinctive condition of the slices obtained (Fig. 10).

\section{Coronal slicing of the fresh cerebral hemisphere}

The following procedural explanation focuses on the option in which the medial aspect of the cerebral hemisphere rests on the movable cutting board (Fig. 8): most of the description is valid for the alternate option, which will be complemented when necessary.

First, the cerebral hemisphere is perfectly aligned by matching the tangent to the ventral aspect of the temporal and occipital lobes with one of the two long edges of the movable cutting board, which are perpendicular to the CIS. Initially, the frontal pole, and then the successive cut surfaces of the cerebral hemisphere are gently and carefully applied against the vertical carpet as shown in Fig. 8. This carpet stabilizes each cut surface of the hemisphere while the sectioning to generate the next slice occurs. The plate with the apposed carpet is perpendicular to the cutting board and serves as a guide for the vertical trajectory of the blade.

To optimize the yield of flawless coronal slices, the following three steps are recommended:

1. Placing the blade of the knife at the site of its entry within the cerebral hemisphere: The cutting edge of the blade that is near the handle of the knife is placed at the precise site where it will enter the hemisphere; the site of entry extends to within $0.5 \mathrm{~cm}$ from the surface of the vertical carpet, the exception being the two adjustments mentioned below to obtain the amygdaloid nucleus or the subthalamic nucleus.

2. Orienting the blade: Once the placement of the blade is made, it is oriented. Ideal orientation requires that:

a. A right angle is formed between the imaginary line of the blade and the long border of the cutting board, which is away from the prosector.

b. The blade is kept perpendicular to the cutting board, and, as mentioned, to within $0.5 \mathrm{~cm}$ from the vertical carpet.

3. The actual sectioning.

As for the brainstem, the cut is performed gently with slow, long strokes, taking advantage of the full length of the blade. The weight of the knife provides most of the vertical force for sectioning. While sectioning, the prosector, using his free hand in an upside-down "U" shaped position, holds the hemisphere as close as possible to the blade of the knife.

Before each cut, the knife is immersed in water, then both sides of the blade are swept with a clean, humid sponge, then sprayed with a mist of absolute alcohol, and then superficially wiped with gauze.

One recalls that the stabilizing carpet is made up of 15 or more layers of gauze. To prevent parenchymal contamination while obtaining the coronal slices, when necessary, one anchor corner of the spoiled layer of gauze is sectioned, and this layer is flipped over to expose the next one beneath it, which is clean. The knife is thoroughly washed and sharpened following each brain processed.

When processing a right cerebral hemisphere, the edge of reference of the cutting board for aligning the tangent to the ventral aspect of the temporal and occipital lobes is the one near the (right-handed) prosector (Fig. 8d). When processing a left cerebral hemisphere, the edge of reference is the one away from the prosector (Fig. 8e). The first cut is $0.5 \mathrm{~cm}$ posterior to the frontal pole. Subsequent slices are made parallel to the first cut at $0.5 \mathrm{~cm}$ intervals. However, to ideally obtain the amygdaloid nucleus (SBB8), a cut should pass through the posterior third of the anterior white commissure (Fig. 10b). To ideally obtain a block of the thalamus including the subthalamic nucleus (SBB17), a cut must pass through the posterior edge of the mammillary body. Thus, the intervals between cuts should be adjusted twice. The hemisphere is sequentially moved toward the CIS, with the cut surface closely applied to it (Fig. 8e).

As the cerebral hemisphere is cut, each slice is placed on a cold metal surface (Fig. 11) with its posterior aspect facing up. They are sequentially laid from left to right, with the first slice (frontal pole) at the upper, left corner of the cold plate. Between 16 and 22 slices are obtained, depending on whether the brain is atrophic or normal, and

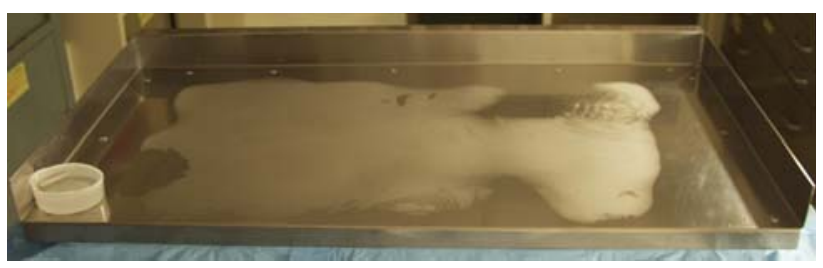

Fig. 11 The cold surface consists of a Styrofoam platform on a mobile cart, which is overlaid by a carpet of ice packs wrapped with a cotton towel on top of which a steel plate is placed. The space between the ice packs and the steel plate can be adjusted to control the temperature of the plate. On the cold steel plate, the coronal slices of the cerebral hemisphere are laid down with the posterior aspect faced up. The container on the left corner of the steel plate holds absolute ethyl alcohol and a razor blade for harvesting blocks from the coronal slices 
whether the donor was brachycephalic or dolichocephalic. The cold surface is used to keep the slices cold during the sectioning of the fresh, cerebral hemisphere, which then, during the harvest of the samples, will be frozen and banked.

\section{Fresh frozen blocks (SBB and ABB)}

We identified 18 brain sites (see below) from which the harvested blocks can match up to $95 \%$ of tissue requests, as per our experience during the past 20 years. The parts


\section{SBB13.1, 13.2, 13.3, 13.4}

Fig. 12 Posterior aspect of fresh, cerebral hemispheric slices, rostral aspect of transverse mesencephalic and myelencephalic slices, and sagittal cerebellar slice. The telencephalic, diencephalic, and cerebellar areas are framed from which the standard brain blocks (SBB) are obtained. The mesencephalon yields two blocks in toto (SBB10.1, 10.2), the myelencephalon two to four (SBB13.1, etc.), as does the metencephalon (not shown) 
of these 18 brain sites can be reliably represented in a series of carefully selected blocks, the harvesting of which we have standardized. Furthermore, the availability of an identical, but contralateral series is crucial for the microscopical assessment and diagnostic categorization of the brains that are banked. Thus, as mentioned, the blocks of this series are referred to as "Standardized Brain Blocks" (SBB); 18 are obtained from the fresh half-brain (Fig. 12), and 18 from the fixed half-brain. Pictures including the anatomical landmarks of the SBB are available online (http://www.newyorkbrainbank.org). The 18 standard brain blocks depicted are the reference blocks (as per their origin and nature) of the SBB series. Because brain tissue is extremely valuable, more blocks are harvested. These extra blocks are either homotopic or heterotopic with reference to the standard series (SBB, see below).

Homotopic extra blocks contain either the same BA or structure(s) as the one(s) included in one of the 18 reference blocks or SBB. For example, SBB1 contains BA9. Many blocks containing BA9 can be obtained. Thus, the additional blocks containing BA9 are homotopic to the reference block SBB1, and are identified as SBB with a denominator that distinguishes each of them (SBB1.2, SBB1.3, etc.), and from the original block or reference block (SBB1.1).

A heterotopic block is a block that does not share its origin with any block of the standard series (SBB) and is referred to as "Additional Brain Block" (ABB), which is further identified according to the BA to which it belongs.

The minimal size of either SBB or ABB is about $0.3 \times 0.5 \times 1.0 \mathrm{~m}$. Their maximal size is about $0.5 \times$ $2.5 \times 3.0 \mathrm{~cm}$.

The series of $\mathrm{SBB}$ and $\mathrm{ABB}$ blocks are removed sequentially from the fresh, coronal slices. To freeze the blocks with liquid nitrogen vapor (LNV) we use a pair of

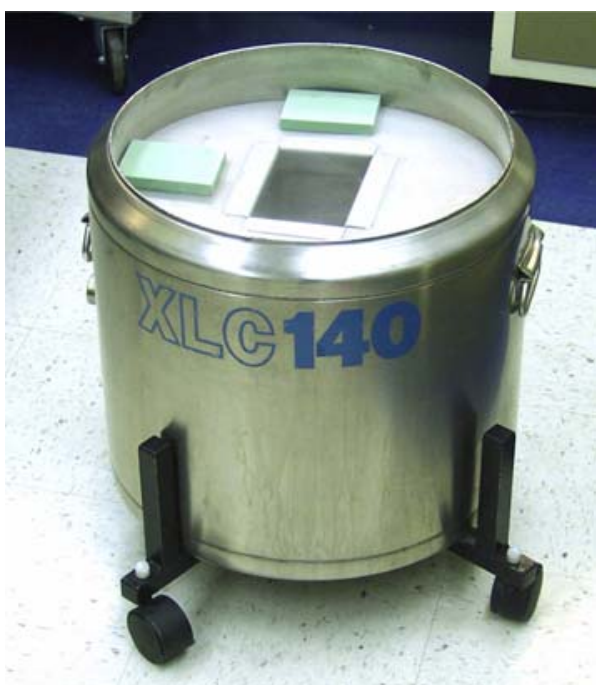

Fig. 13 Vessel model XLC140, and a pair of Teflon-coated aluminum plates. A pair of Teflon-coated, aluminum plates (each plate measuring $1.0 \times 8.0 \times 10.0 \mathrm{~cm}$ ) for freezing blocks of tissue, rest on the shoulder of the double-walled vacuum vessel XLC140 (XLC140 vessel provider (8/2002): LNV Freezing Vessel, MVE, Inc., Two Appletree Square, Suite 100, 8011 34th Avenue South, Bloomington, MN 55425-1636. Phone: 1-800-2474446, ask for Steve Shaw, Sales Manager. XLC140 vessel with plain lid cover, foam insulation, vapor shipper insert, and caster wheelbase. Ask for Harvard BTRC specifications.)

Teflon-coated aluminum plates (each plate measuring $1.0 \times 8.0 \times 10.0 \mathrm{~cm}$ ) and the XLC140 vessel (Fig. 13).

To obtain LNV, we use an XLC140 vessel (Fig. 13). The walls of this vessel include a sponge that absorbs liquid nitrogen (LN) and gradually releases it as vapor or LNV within a centrally located cavity where the samples are being frozen. Liquid nitrogen vapor allows freezing samples at $\mathrm{LN}$ temperatures without $\mathrm{LN}$ contact. Because LNV as compared to LN exerts less stress on the tissue, and minimizes the interface during the freezing process,
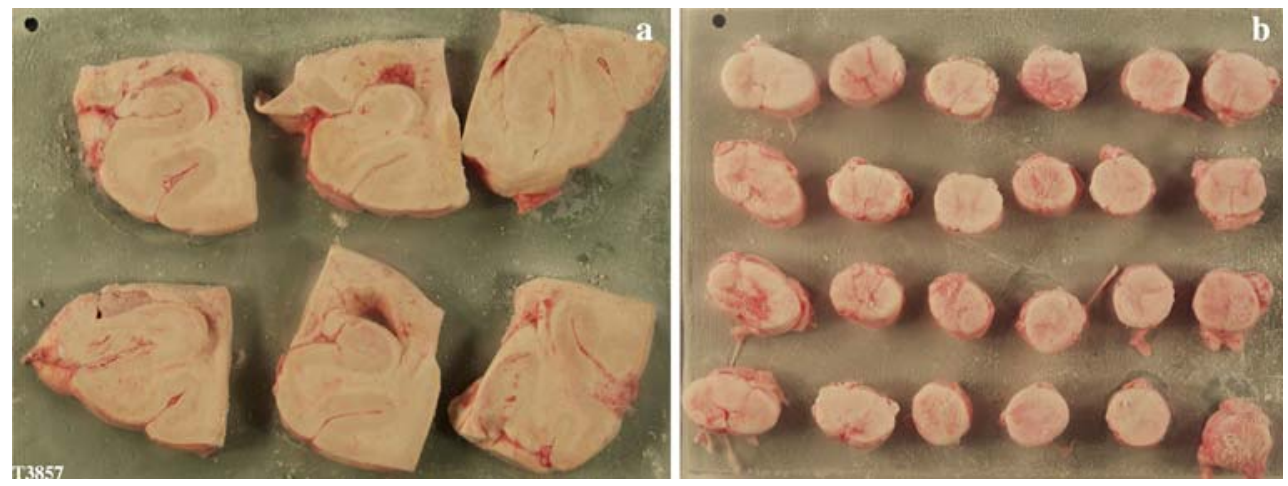

Fig. 14 Lower Teflon-coated aluminum plate with fresh frozen samples, as they appear upon removal of the upper Teflon-coated aluminum plate. Five levels of the frozen hippocampal formation
(SBB5, body; SBB18, head of hippocampus) were obtained from one half-brain (a). Lower plate with 24 alternate frozen segments of the spinal cord (b) 
XLC140 vessel (or an equivalent vessel) is crucial for the processing of a brain for research, yielding blocks with minimal or even without freezing artifacts. At regular intervals (once/24 h), LN is added to the XLC140 vessel to maintain its low temperature $\left(-160\right.$ to $-180^{\circ} \mathrm{C}$; the actual temperature of liquid nitrogen is $-195^{\circ} \mathrm{C}$ ). $\mathrm{LN}$ is stored in a special tank in the dissecting room. A backup tank of $\mathrm{LN}$ is kept available.

The pair of pre-cooled $\left(-70\right.$ to $\left.-100^{\circ} \mathrm{C}\right)$ Teflon-coated aluminum plates is used to create a "sandwich". The "sandwich" consists of a lower plate on which five or more fresh blocks are laid flat (Fig. 14). Then, on top of the blocks, the upper plate is gently apposed. Next, the "sandwich" is instantly transferred within the cavity of the XLC140 vessel. A lid is placed on top of the XLC140 vessels while the freezing takes place (about $5 \mathrm{~min}$ ). Once frozen, the blocks are removed from the "sandwich", and carefully arranged on the cold shoulder of the XLC140 vessel in such a way as to avoid mixing up the blocks. Next, each block and its specific barcode label are transferred into an individual plastic bag, which is then sealed (Fig. 3). The bagged samples are subsequently stored temporarily at $-80^{\circ} \mathrm{C}$ in an operational freezer and then electronically tracked and dispersed in storage freezers (see below).

\section{Fresh frozen brain parenchyma aliquots}

In addition to both the SBB and $\mathrm{ABB}$ series, samples of cerebral cortex, cerebral white matter, and cerebellum are obtained separately to provide aliquots of fresh frozen parenchyma. These aliquots are ideal for biochemistry, protein studies, or molecular biology investigations. They are not suitable for studies requiring the preservation of cytoarchitecture or cellular morphology. The process of freezing these cerebral or cerebellar cortical aliquots differs from the process of freezing the white matter aliquots.

\section{Cerebral or cerebellar cortical aliquots}

Once the SBB and ABB blocks have been obtained, fresh blocks of cerebral or cerebellar cortexes are harvested from the leftovers of the slices. When applicable, these samples are obtained from defined BA as accurately as possible.

The cortical samples of either the cerebrum or cerebellum are immersed in liquid nitrogen, and then pulverized in a porcelain mortar. The resulting granules are transferred into $2.0 \mathrm{ml}$, polypropylene "Wheaton Cryule, $2.0 \mathrm{ml}$ " vials [Fisher catalog 03-341-18H, E, G (Fig. 3)]. To maximize the anatomic specificity, both the mortar and

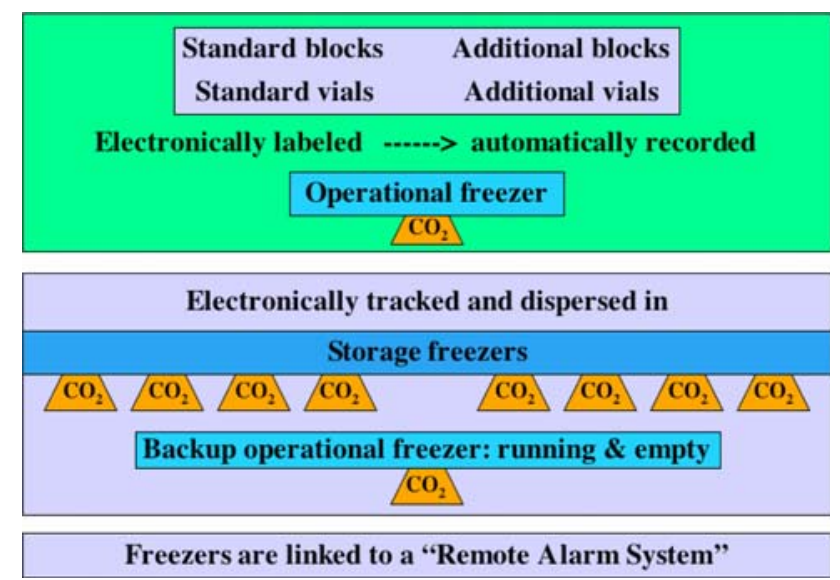

Fig. 15 Operational and storage freezers; sample dispersal, $\mathrm{CO}_{2}$ backups, and alarm. Each freezer is connected to: (1) the standard hospital electric supply network; (2) the emergency generator dependent electrical supply network; (3) a $\mathrm{CO}_{2}$ cylinder; and (4) remote alarm system. An extra backup set of $\mathrm{CO}_{2}$ cylinders is kept in the storage freezer room

pestle must be carefully cleaned before processing samples from each of the different regions.

Cerebral white matter aliquots

The white matter samples are obtained from the center semiovale and consist of cores measuring about $3.0 \times$ $0.3 \times 0.2 \mathrm{~cm}$. The cores are laid down flat on a pre-cooled, Teflon-coated, $1.0 \times 8.0 \times 10.0 \mathrm{~cm}$ aluminum plate, and then transferred into the XLC140 vessel. Next, the appropriately labeled vials are filled with these frozen cores.

\section{Freezers}

We use "ultra-low freezers" $\left(<-70^{\circ} \mathrm{C}\right)$ grouped into two categories (Fig. 15):

\section{Operational freezers and storage freezers}

Each freezer is connected to: (1) the standard, hospital electric supply network; (2) the emergency generator dependent electrical supply network; (3) $\mathrm{CO}_{2}$ cylinders; and (4) remote calling alarm system.

\section{Operational freezers}

Two operational freezers are used. "Freezer A" is located within the dedicated brain cutting room, and "Freezer B" is kept within the freezer storage room. 
"Freezer A" is used for temporary storage of the frozen samples obtained at the time of processing the fresh brain for research. Samples in "Freezer A" are electronically labeled, but not tracked. Once a week, the newly acquired, barcode labeled, and frozen samples are removed from "Freezer A" and electronically tracked while dispersed within the storage freezers (see below).

"Freezer B" is the backup freezer. This freezer is to be used exclusively for emergency situations, and only during the period required for repairing or replacing the disabled storage freezer. Thus, "Freezer B" must be kept empty and running so that frozen samples can be transferred and stored in it temporarily, should one of the storage freezers fail. In case of a freezer failure, the layout of any storage freezer can be replicated in "Freezer B" without disrupting the electronic tracking coordinates of the samples provisionally kept in it.

\section{Storage freezers}

Storage freezers are kept in a dedicated room and used for storing electronically tracked, frozen samples. These samples are made available for research, locked, or kept indefinitely as DNA reservoirs, which are duplicated and stored in two separate freezers.

Briefly, the cabinet space of the storage freezers is compartmentalized hierarchically into shelves, racks, columns, and boxes. Two groups of boxes are used: (1) for storing the LNV-frozen, standardized brain blocks (SBB)
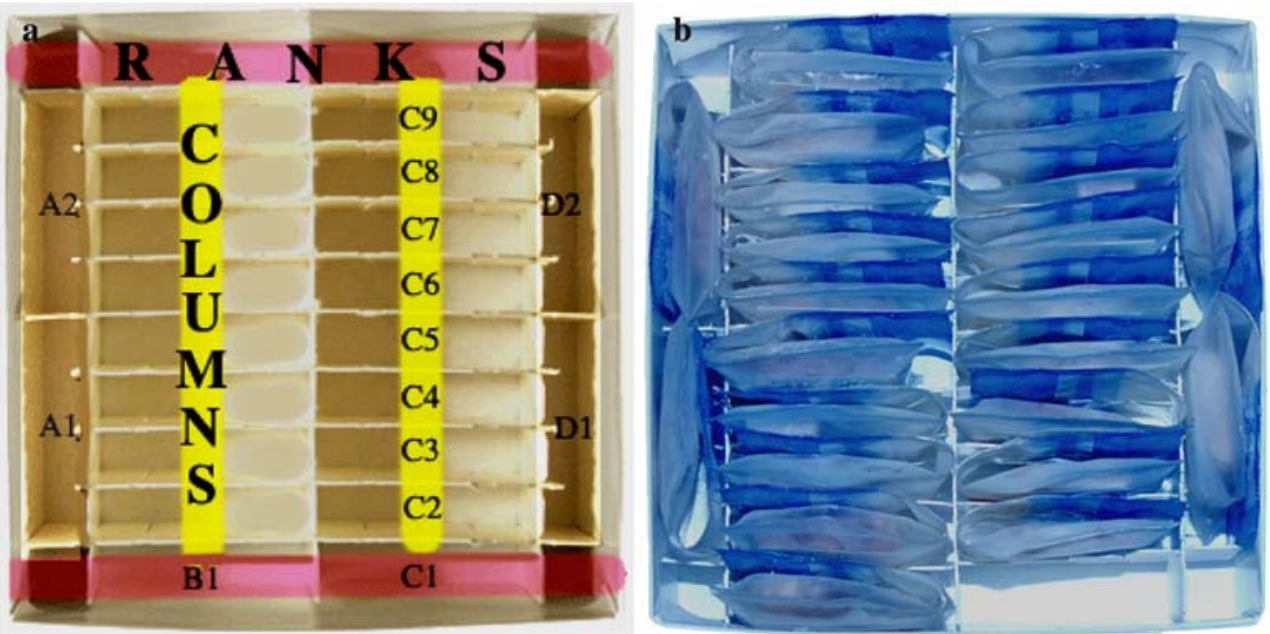

Fig. 16 Empty SBB, or ABB, or block "box-area" (Fiberboard Storage Box, Fisher catalogue 5954, divider 11-678-24C) (a). Partially filled $\mathrm{SBB}$, or ABB, or block "box-area" (b)

Fig. 17 Empty vial "box-area" (Fiberboard Storage Box, Fisher catalogue 5954, divider 13-989218) (a). Filled vial "box-area." The filled vial "box-area" contains 81 vials, each of which is electronically tracked (b)
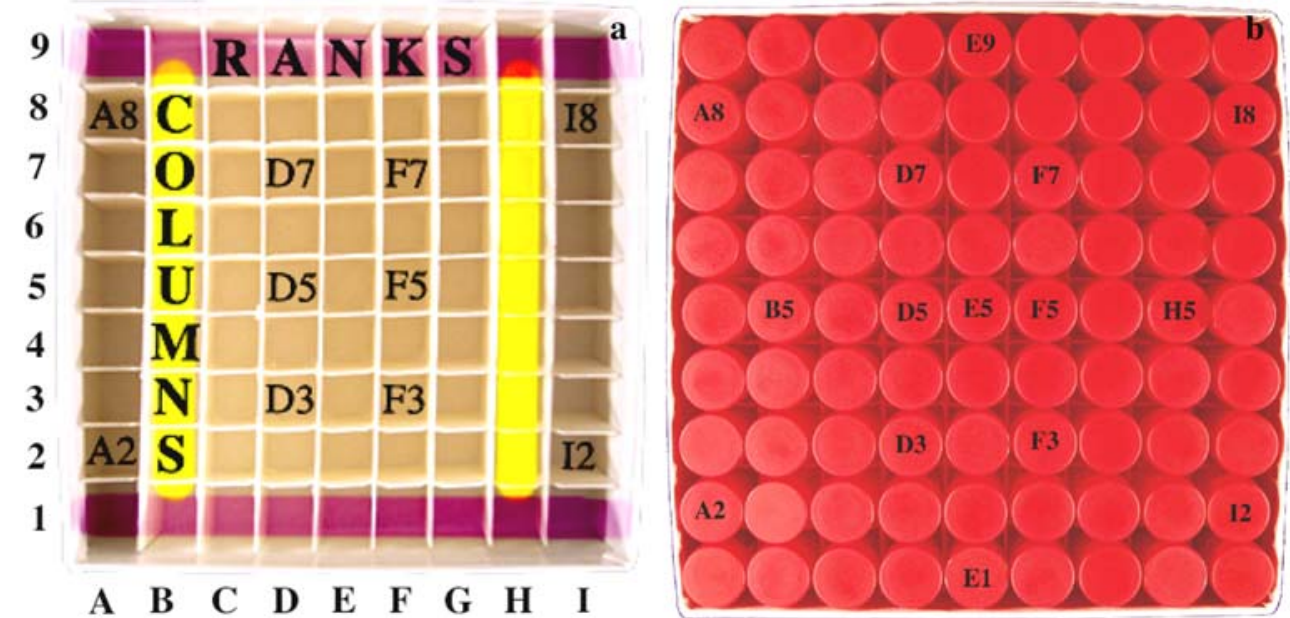
and additional brain blocks (ABB) (Fig. 16); and (2) as boxes for samples included in vials (brain aliquots) (Fig. 17). A barcode label uniquely defines each freezer, shelf, rack, or box, in addition to each sample that is harvested for research and stored.

\section{Box-area versus box-brain}

The most important compartment of a storage freezer is the box in which the samples are kept (Figs. 16, 17). Traditionally, a dedicated freezer space or box, which is hereafter referred to as "box-brain," was assigned to a brain (case). Now, the storage of samples is based on the concept of "box-area" instead of "box-brain." The numerical identifier of the brain determines the identity of the "box-brain" where it is stored, while the anatomical origin of the samples determines the identifier of the "box-area", as "area" refers to the anatomical origin of the samples the box contains. SBB containing the same anatomical structures are stored together, independent of their source brains. They are kept in boxes (SBB or ABB "box-area"), the designation of which depends on the anatomical characteristics of the blocks they harbor (Fig. 16). Similarly, vials containing either cortical or white matter aliquots from the identically targeted BA are stored together independent of the brain from which they were obtained. They are kept in a vial "box-area" (Fig. 17).

A SBB "box-area" has 24 compartments (Fig. 16a). A filled "box-area" contains 24 LNV-frozen blocks, all including the same anatomical area from up to 24 brains (or 24 source brains). Only 23 compartments are occupied in the box depicted in Fig. 16b. One compartment (lower right) is free, and is recorded as such by the software. This compartment will be replenished when the next series of samples will be stored. Overall, this electronic tracking of the compartments that become free upon ongoing disbursements of samples enhances the use of the freezer cabinets by $45 \%$.

A vial "box-area" has 81 compartments (Fig. 17). Thus, a SBB or a vial "box-area" contains samples from the same anatomical area, which were obtained from many brains. To ease the location of a sample in either SBB "box-area" or vial "box-area," the site of each of the 24 or 81 compartments is defined through coordinates. The algebraic system used includes "columns", and "ranks" or "rows" (Figs. 16, 17).

Storing samples using "box-area" rather than "boxbrain" minimizes the dead space in freezers that results when specific parts of a brain are disbursed. Thus, a given "box-area" can be replenished proportional to the number of samples retrieved for disbursement. This necessitates a rigorous monitoring of sample inputs and outputs. In contrast, free space resulting from disbursements of parts of a brain stored in a "box-brain" cannot be re-used because the identifiers of both the "boxbrain", and the brain the "box-brain" contains, are shared. Retrieval of samples is faster if stored in "boxarea" rather than "box-brain", for most requests include the same areas from many source brains. Thus, the time during which a freezer is kept open for the retrieval of samples can be reduced, which in turn decreases the fluctuations of its cabinet temperature and hence the stress on the tissue samples.

\section{Dispersal of samples in storage freezers}

The "box-areas" are stored in such a way that samples of a single brain are dispersed in 3-6 separate storage freezers, depending on the number of samples obtained from the source brain. Thus, in the event of a failure of an individual freezer, despite the provisions in place, it is unlikely that the entire set of samples obtained from a single brain would be ruined.

Samples cannot be added or retrieved from any storage freezers without the supervision of the laboratory supervisor, except in emergency situations. For example, if a storage freezer fails, whoever happens to be in a position to perform the transfer of samples from the idle freezer to the backup operational freezer will perform the transfer. If such a transfer must be undertaken, the following precautions must be taken. While transferring the racks, one must make sure that the topographic status or distribution of these racks in the back-up freezer matches that of the disabled freezer. This precaution keeps valid the coordinates of the electronic tracking of the samples that must be transferred and stored temporarily in the backup operational freezer.

\section{Assessment of tissue quality (PMI, pH, ribosomal RNAs)}

Antemortem events, such as anoxia or hypovolemia, affect the molecules in brains more profoundly than a prolonged postmortem interval at least up to $24 \mathrm{~h}[3,15$, $32,34]$. There is no significant change in the $\mathrm{pH}$ of the CSF or of brain samples within a postmortem interval of $24 \mathrm{~h}$ [3].

Measurement of tissue $\mathrm{pH}$ provides a means to screen a postmortem brain for RNA preservation, and helps match control versus diseased brain samples [5, 32, 40].

Qualitative assessment of ribosomal RNA, and determination of tissue $\mathrm{pH}$ are performed using two blocks (one 
for RNA and one for $\mathrm{pH}$ evaluations, each block measuring $0.5 \times 0.5 \times 1.0 \mathrm{~cm})$. These blocks are harvested at the preoccipital notch (BA37), where the watershed territory of the anterior, middle, and posterior cerebral arteries is located. If a longstanding, terminal hypoxia or hypovolemia occurred, then, theoretically, related changes involving this site should be reliably representative for the rest of the brain. Harvesting of these two blocks is performed at the end of the brain processing to maximize the accuracy of the assessment of tissue quality.

\section{Postmortem interval (PMI) determination}

The lapse of time between the transition from life to death (or mortal agony) can be short or protracted with or without metabolic imbalance, which inevitably affects the quality of the brain and the onset of autolysis [1, 17, 22, $23,29,35,41,44]$. Autolysis of the brain is believed to begin with the occurrence of death, the exact time of which may or may not be determined precisely. The influence that autolysis has on the quality of brain samples prepared and used for research is multifactorial and unpredictable $[2,12,14,48]$. Some investigators opine that the effects of autolysis are minimal within $5-12 \mathrm{~h}$ or even $24 \mathrm{~h}$ or longer following death [4, 6, 36, 42, 50]. Experiments assessing the effects of the postmortem intervals on brains using mice or rats support these observations [38]. Nonetheless, to minimize the conjectural effects of autolysis, the preparation of samples for research at the NYBB is performed as soon as possible after death, on a $24 \mathrm{~h} / 7$ days a week schedule.

There are no universally accepted criteria for defining the postmortem interval (PMI) [52]. To gain insight about the effect of autolysis on tissue quality, two postmortem intervals are recorded. One is referred to as "postmortem interval-cold room" (PMI-cr). The other is referred to as "postmortem interval-frozen" (PMI-fzn), which is more or less equivalent to the PMI commonly referred to in the scientific literature.

- PMI-cr is computed according to the following formula:

PMI-cr(in hours)

$=$ Time when the body or brain is put in cold $(*)$

- Time of death

*: "In cold" means when (date including hour) the body is placed in a cold room [usually with ambient temperature ranging between 5 and $10^{\circ} \mathrm{C}$; or when (date including hour) the brain is removed, and placed on wet ice].

- PMI-fzn (or PMI) is computed according to the following formula:

PMI-fzn(in hours)

$$
\begin{aligned}
= & \text { PMI }=(\text { Time starting processing }(*)+1 \mathrm{~h}) \\
& - \text { Time of death }
\end{aligned}
$$

*: Time starting processing is the time when the processing of Protocol 1 starts. $1 \mathrm{~h}$ is added to this time to include the
Fig. 18 Cerebral cortex [SBB4, Brodmann area 18; 60-year-old man, control; postmortem interval frozen (PMI-fzn): 14:30 hours] frozen with liquid nitrogen vapor. The corticosubcortical junction is at the bottom of the picture (a). The field magnified (b) is from an area located near the center of $\mathbf{a}$, just to the left of the V-shaped vessel. The morphology of neurons, astrocytes, or oligodendrocytes, is preserved. A $10 \mu \mathrm{m}$ thick section. HE



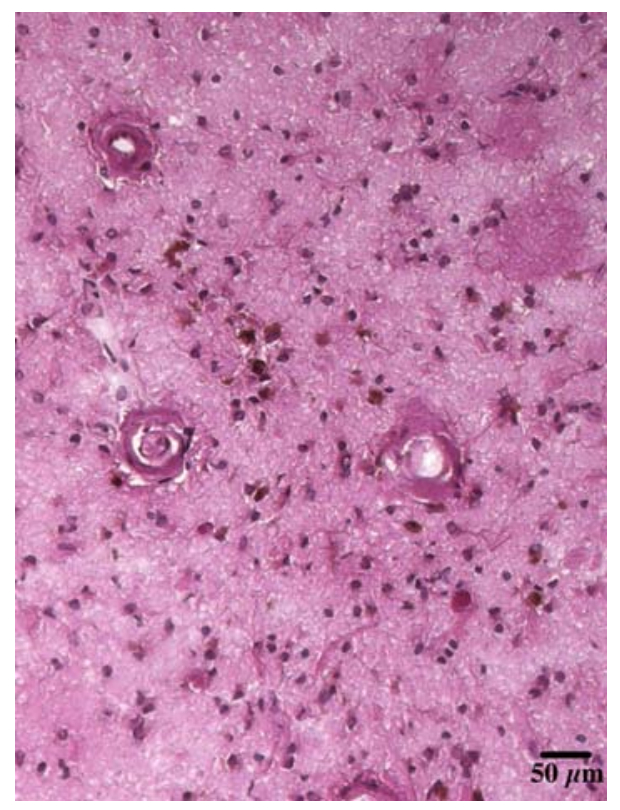

Fig. 19 Cerebral cortex [SBB3, Brodmann area 7; 91-year-old demented woman with multiple cerebral hemorrhages; postmortem interval frozen (PMI-fzn): 17:45 hours] frozen with liquid nitrogen vapor. Freezing artifacts are minimal despite the poor condition of the brain in the fresh state. Cerebral amyloid angiopathy, hemosiderinophages, gliosis, neuritic plaques, and neuronal loss are clearly identifiable. A $10 \mu \mathrm{m}$ thick section. HE

lapse of time needed for harvesting and freezing the samples.

\section{$\mathrm{pH}$ determination}

The block of frozen cerebral cortex (BA37) is weighed. Water at neutral $\mathrm{pH}$ is added to the tissue $(1.0 \mathrm{ml} / 100.0 \mathrm{mg}$ of tissue), followed by thorough homogenization with a tissue homogenizer. $\mathrm{pH}$ values are measured in triplicate and at room temperature, using a standard Corning electrode.

\section{RNA assessment}

The quality of $28 \mathrm{~S}$ and $18 \mathrm{~S}$ ribosomal RNAs of each brain prepared for research is assessed. Briefly, total RNA is extracted from $50.0-100.0 \mathrm{mg}$ of cerebral cortex (BA37) using RNA-STAT 60 reagent (Tel-Test, Inc., 1511 Country Road 129, Friendswood, TX 77546). Degradation is then tested with electrophoresis, using $1 \%$ agarose gel. The bands are stained with ethidium bromide and photographed digitally. A picture of the RNA gel and the $\mathrm{pH}$ values are included in the anonymous file of the corresponding brain.

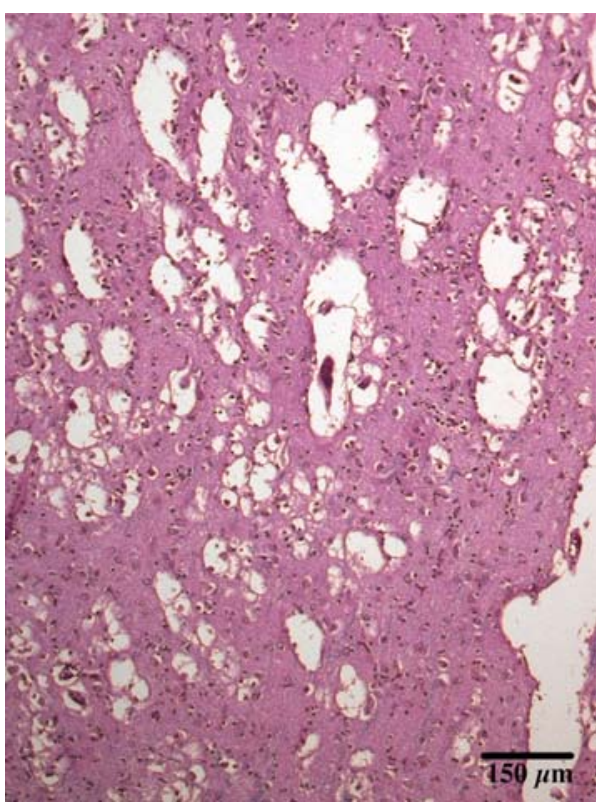

Fig. 20 Cerebral cortex [SBB4, Brodmann area 18; 92-year-old demented woman; postmortem interval frozen (PMI-fzn): 03:55 hours] frozen with dry ice. Marked ice-artifacts; however, cellular identification is still possible. A $10 \mu \mathrm{m}$ thick section. LHE

Our current method for assessing the $\mathrm{pH}$ is probably less reliable than using a "Bioanalyzer", which we will acquire in the near future.

Freezing artifacts

As mentioned, LNV is used as a cryogen, which allows freezing samples at $\mathrm{LN}$ temperature (about $-180^{\circ} \mathrm{C}$ ) without LN contact. The interface between the brain samples (the temperature of which is about $5-10^{\circ} \mathrm{C}$, or more) and $\mathrm{N}$ vapor is minimized. Brain blocks measuring up to $0.5 \times 2.5 \times 3.0 \mathrm{~cm}$ remain intact when frozen with the vapor phase of N. In contrast, they tend to fragment into pieces when frozen with the liquefied phase of $\mathrm{N}$. Furthermore, with LNV, freezing artifacts are minimal (Fig. 18a). The morphology of neuron, astrocytes or oligodendrocytes is preserved (Fig. 18b). Many pathological changes can be readily identified (Fig. 19). Notably, histology sections from large blocks, which were frozen using LNV, show less freezing artifacts than sections obtained from blocks frozen on dry ice with its enthalpy of sublimation at $-78^{\circ} \mathrm{C}$ (Fig. 20). The table lists major pros and cons between the latest methods developed using LNV and those using dry ice, either by freezing half-brain en bloc, or first by obtaining slices at the fresh state, which are then frozen on dry ice (Table 1). 
Table 1 Comparison of freezing methods

\begin{tabular}{lll}
\hline Condition & Advantages & Disadvantages \\
\hline Frozen "En bloc", usually dry ice $\left(-78.5^{\circ} \mathrm{C}\right)$ & $\begin{array}{c}\text { Freezing does not require expertise or } \\
\text { special apparatus, is simple and quick; } \\
\text { entire half brain is banked }\end{array}$ & $\begin{array}{l}\text { Evaluation of tissue confined to external } \\
\text { surface; large space in freezer; difficult } \\
\text { to access within freezers; must be } \\
\text { warmed up to access sites } \rightarrow \mathrm{T}\end{array}$ \\
& & $\begin{array}{l}\text { variations; harvest of areas difficult, } \\
\text { dangerous; anatomical origin often } \\
\text { questionable; inter-regional } \\
\text { parenchyma contamination; severe }\end{array}$ \\
& & $\begin{array}{l}\text { ice artifacts } \\
\end{array}$ \\
& &
\end{tabular}

Sectioned fresh slices $\rightarrow$ dry ice $\left(-78.5^{\circ} \mathrm{C}\right)$

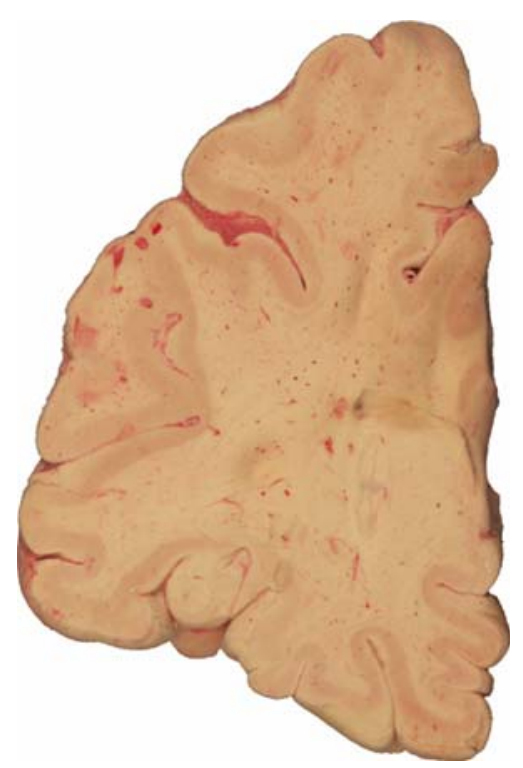

NYBB present protocol (LNV: $\left.-195^{\circ} \mathrm{C}\right)$

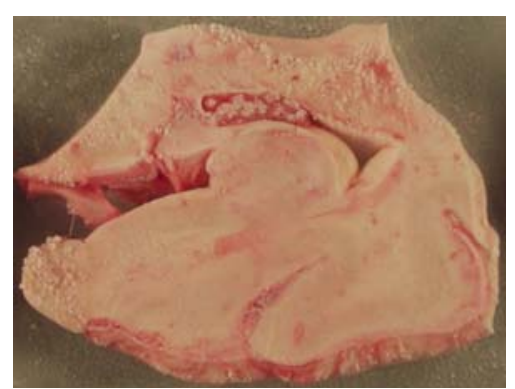

Thorough gross evaluation of tissue; minimal instrumentation; easy access to any part of interest, e.g., head of hippocampus, pulvinar, etc.; moderate freezing artifacts
Irregular slices unless performed by competent prosectors; often retrieval of many slices to find the exact target; slice is warmed up to harvest area of interest $\rightarrow \mathrm{T}$ fluctuations; monitoring the leftover is cumbersome, which complicates further assessments of eligibility for fulfilling requests
Thorough gross evaluation. Quick disbursement; precise anatomy, no fluctuation of T, easy to store and track; mild freezing artifacts; increased freezer efficiency
Laborious dissection usually including two individuals, and at least one with good command of the protocol, anatomy, and neuropathology; special apparatus, dedicated space for processing
Thin sections (7-14 $\mu \mathrm{m}$ thick) obtained from an optimally frozen block may be studded with unexpected vacuoles. Thus, these vacuoles may not be caused by the freezing of the block at the time of processing the fresh brain. However, improper handling of blocks, or of the individual thin sections while generating them within the cryostat, or during the staining procedure, may produce confounding artifacts (Fig. 21a, b). As a rule, a block, 


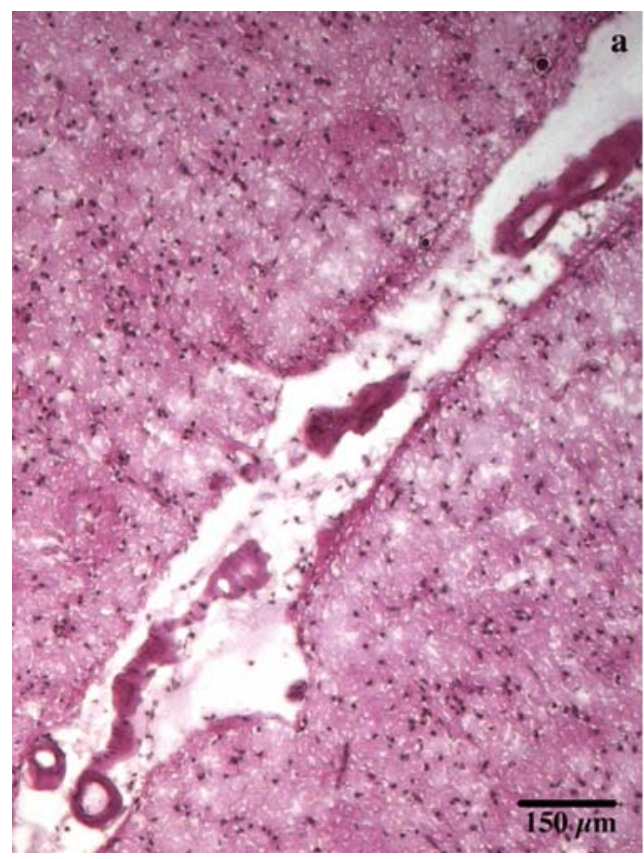

Fig. 21 Serial, $10 \mu \mathrm{m}$ thick sections from the cerebral cortex (same patient as in Fig. 19) without (a) or with (b) prominent artifacts, which consist of innumerable, optically empty vacuoles. These vacuoles are not due to the original freezing of the block, as they are

which is stored at $-80^{\circ} \mathrm{C}$, should be warmed-up at $-20^{\circ} \mathrm{C}$ overnight before thin cryo-sections are being produced. These sections are fragile and hence they must be manipulated with extreme care. Placing the thin sections on slides must be done carefully without stretching the parenchyma. Then once, one or more sections are attached to the slide, the complex slide-tissue must be immediately immersed in the fixative of choice. It is imperative to avoid dehydration of the sections. Dehydration causes extensive vacuolization of the parenchyma. These vacuoles mimic the tears caused by the formation of intraparenchymal crystals of ice, which occur when the freezing of blocks is too slow because the temperature is not cold enough. The formation of intraparenchymal crystals of ice can be more efficiently averted by using, for example, LNV instead of dry ice.

\section{Diagnostic categorization and standardized neuropathologic evaluation}

Clinical and pathologic data determine the categorization of the brain for research. Thorough, standardized pathologic examination is performed to give the best survey of normal or abnormal brains. For the neuropathological evaluation, the contralateral half-brain is used, immersed in 2.01 buffered, $10 \%$ formalin solution, at room temperature, over 7-10 days.

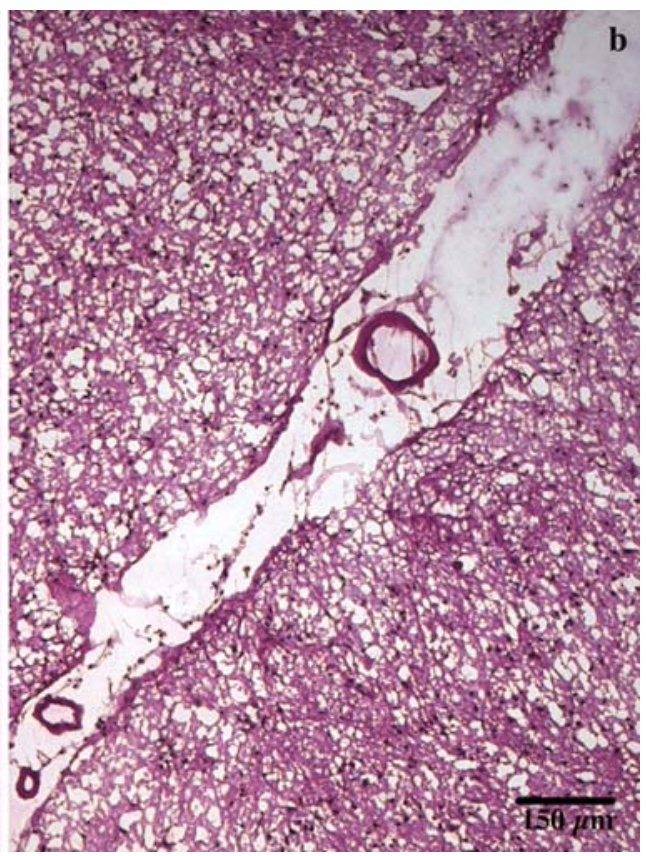

not seen in a or in Fig. 19, but secondary to dehydration that occurred during the interval of time that the thin section was laid on the slide and immersed in absolute alcohol. HE

\section{Macroscopic examination}

The weight of the fixed half-brain is recorded. Pictures are taken. Vascular stenosis and residual lumen are estimated and recorded as a percentage. The cerebellum and brainstem are separated from the cerebral hemisphere according to the methods used for the half-brain set aside for research (see above). The cerebral hemisphere is sectioned coronally on a set of standard landmarks with extra cuts in between, in front, and behind the landmarks, according to the size of the brain (30-35 slices).

The landmarks are: Immediately anterior to the temporal tip, anterior margin of the optic chiasma, $3.0 \mathrm{~mm}$ posterior to the anterior commissure (to obtain the amygdaloid nucleus, all slices are parallel to this line), infundibulum, anterior third of the mammillary body and $3.0 \mathrm{~mm}$ posterior to mammillary body (to cut through the subthalamic nucleus-in contrast, when processing the fresh half-brain, the cut grazes the posterior edge of the mammillary body to obtain a block that includes the subthalamic nucleus), midpoint of cerebral peduncle, posterior junction of peduncle with midbrain tegmentum (to obtain lateral geniculate body).

The first cut passes $3.0 \mathrm{~mm}$ caudal to the cut surface of the anterior commissure on the medial aspect of the cerebral hemisphere. This cut is in an axis perpendicular to a line that is tangential to the ventral aspect of the inferior temporal gyrus and occipital pole. This cut provides both 
the internal and external segments of the globus pallidus, and all slices are cut parallel to this section. The brainstem is sectioned transversely, and the cerebellum is sectioned sagittally, each at $0.3 \mathrm{~cm}$ intervals.

Any atrophy is graded from 0 (no atrophy) to 4 (very severe atrophy). Focal lesions are described by size, shape, contour, color, consistency, side, and site (SS CCC SS).

\section{Microscopic examination}

As stated, 18 standardized, representative blocks are selected for microscopic examination (Fig. 22). The standardization of the blocks is an integrative component of the brain bank operation (Figs. 2, 4, 5, 10, 12, 14). These blocks closely match those harvested from the contralateral half-brain, which are prepared for research (Fig. 12). Thus, paraffin block no. 1 of the fixed half-brain matches LNVfrozen block no. 1 (SBB1), etc.

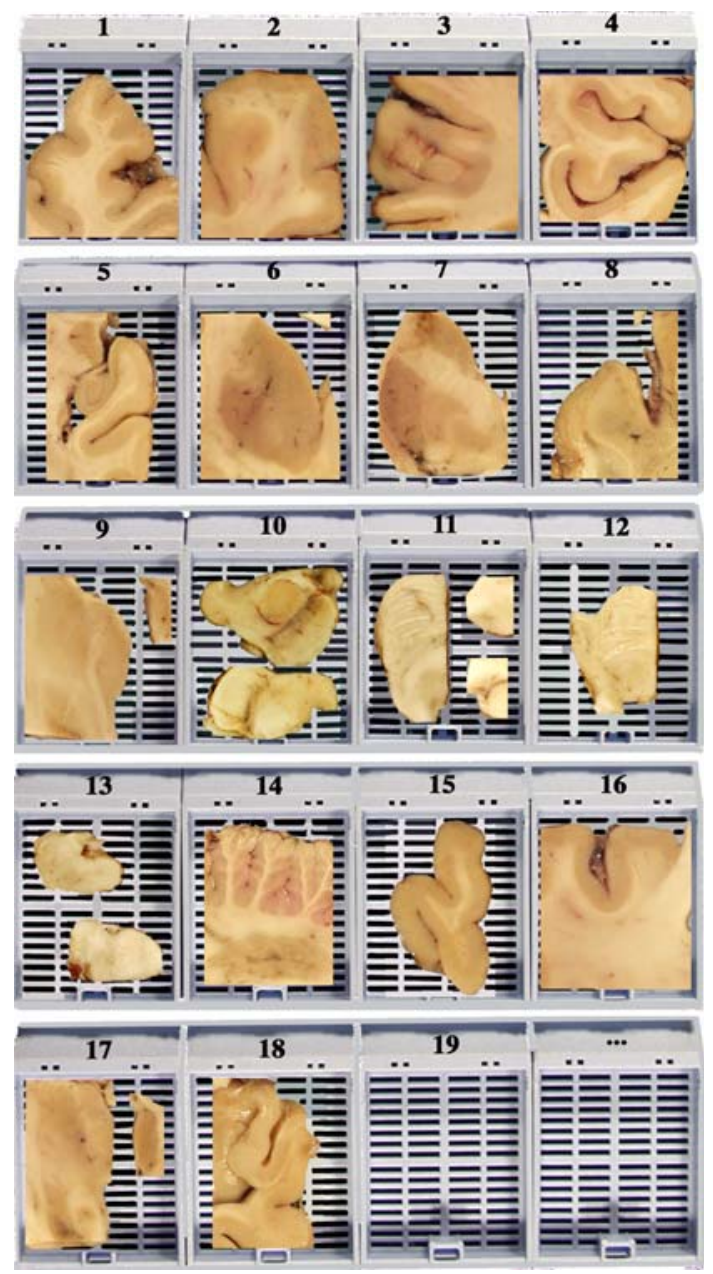

Fig. 22 Eighteen standardized blocks harvested for microscopic examination. This series matches the standardized series obtained from the contralateral half-brain prepared for research, which are referred to as standard brain blocks (SBB)
Additional blocks are taken when directed by the gross examination of the specimen or by clinical history. There is representative, histological documentation of the lesions identified grossly. Paraffin sections with a thickness of $7 \mu \mathrm{m}$ from all blocks are stained with Luxol-fast-blue and counterstained with hematoxylin and eosin for general survey. Selected sections are stained with Bielschowsky for evaluation of axons, neuritic plaques, and neurofibrillary and glial tangles; antibodies against $\beta$-amyloid, for vascular and parenchymal deposits; phosphorylated tau for neuronal and glial tangles; ubiquitin for ubiquitinated cytoplasmic, nuclear, or axonal aggregates; $\alpha$-synuclein for Lewy bodies, Lewy neurites, and glial tangles; or other antibodies as indicated by findings or history. The mean number of neuritic plaques in five, random, $100 \times$ fields per slide is recorded using Bielschowsky-stained slides or $\beta$-amyloid labeled sections from blocks $1,2,3,4,5,8$, and 18 .

The leftovers of every brain banked are placed in formalin in a separate, dedicated container for each half, which carries a color-coded label with an anonymized identifier. The containers are kept in the storage room for formalin-fixed samples of the NYBB. The histology slides are filed in cabinets that are kept within the laboratory of the NYBB for immediate access when necessary. The formalin phosphate fixed and paraffin embedded blocks are stored within the facilities of the Department of Pathology and Neuropathology of the New York Presbyterian Hospital, as the NYBB is an integrated part of this Department. Thus, the rest of the fixed half-brain and the leftover of the contralateral half, the paraffin blocks, and histologic sections are stored and made available for further diagnostic procedures when indicated or for additional investigations (e.g., comparative, morphological, or quantitative studies).

\section{Neuropathology reports and diagnostic categorizations}

The gross and microscopic examination results are recorded in two standardized, neuropathology reports for each brain. One report is narrative or text-based as part of the patient's chart. The second report is electronic, including semi-quantitative assessments of changes, to identify samples stored for research according to criteria such as the extent of degenerative changes.

For the diagnostic categorization of the brains, we use the published criteria listed on the NYBB web site (http:// www.newyorkbrainbank.org). For example, we assign the diagnosis of Alzheimer disease when the criteria of "The Consortium to Establish a Registry for Alzheimer's Disease" (CERAD) are met [33]. The likelihood that dementia is due to the Alzheimer changes (neuronal loss, presence of neurofibrillary tangles of Alzheimer and of neuritic plaques) is assessed according to the criteria proposed by 
"The National Institute on Aging, and Reagan Institute Working Group on Diagnostic Criteria for the Neuropathologic Assessment of Alzheimer's Disease" [46]. Furthermore, we assign a Braak and Braak stage to reflect the extent of involvement of the neurofibrillary tangles of Alzheimer [8, 9] or that of the Lewy body-containing neurons [10]. A brain is assigned to the category of Alzheimer disease Lewy body variant (ADLBV) if there is documented dementia, neuronal loss with neuritic plaques, and neurofibrillary tangles that occur in numbers of diagnostic significance for $\mathrm{AD}$, and with cortical and subcortical Lewy bodies [19, 20]. The subcortical areas with Lewy bodies include substantia innominata, amygdala, hypothalamus, substantia nigra pars compacta, nucleus coeruleus, and dorsal nucleus of vagus. These criteria are subject to changes contingent to authoritative guidelines.

\section{“Distributive Diagnoses" versus "Request Diagnoses"}

To identify samples in storage that best match the specific requirements of an investigation, a link combines the file of requests (filled by the investigator) with the file including the characteristics of the samples in storage (filled by the neuropathologist). The request file lists a set of questions addressed to the investigators; familiarity with either the neuroanatomy or neuropathology, or both, may vary. The questions guide the requestors; the answers greatly contribute to the pre-selection of the pool of samples that may or may not be eligible for a particular study. In addition, the request file offers a set of pictures providing details about the neuroanatomy and the SBB with their structures or areas, which are labeled.

The quintessence of the categorization of these samples is the "distributive diagnosis", which is assigned to each brain and, by extrapolation, to each of its derived samples. Furthermore, a "matching diagnosis" is assigned to each brain, which is a more general diagnostic subgroup, and thus less specific than the "distributive diagnosis". The subgroup "matching diagnosis" allows an electronic-supported, pre-selection of brain samples that may be suitable for a given request. Both the clinical (CDx) and the neuropathologic diagnoses (NPDx) determine the distributive diagnosis (DDx) of any brain processed and made available for research. Thus, the summary formula is CDx + NPDx = DDx. For example, if the thorough macroscopic and microscopic examinations using conventional methods of brain evaluation do not reveal any specific diagnostic abnormality, the neuropathologic diagnosis assigned to this brain would be "Diagnosis: There is no diagnostic abnormality recognized". Furthermore, if the clinical diagnosis is "myocardial infarct" and the individual were without any neurological or neuropsychiatric impairment, the distributive diagnosis would be "No diagnostic abnormality recognized" and the matching diagnosis would be "Normal control." However, if the clinical diagnosis were "Schizophrenia", then the distributive diagnosis would be "No diagnostic abnormality recognized-Schizophrenia", and the matching diagnosis would be "Schizophrenia". Thus, this brain would be suitable for research on schizophrenia. That, in schizophrenia, volumetric loss of the brain or enlarged ventricles compared to individuals without neurologic or psychiatric disorders occurs, or that changes involving the pyramidal cells of the entorhinal cortex may be detectable using conventional methods of neuropathological evaluation, although not specific, would further support the eligibility of this brain for studies on schizophrenia $[21,26]$.

Brains from individuals without neurologic or psychiatric disorders may or may not have focal lesions. When no lesion is found on thorough neuropathologic examination, the distributive diagnosis assigned is "No diagnostic abnormality recognized" and the matching diagnosis is "Normal control." However, if asymptomatic infarcts, or sequelae of a subacute or old trauma, or acute, focal damage involving an otherwise unremarkable brain were found on examination, the distributive and matching diagnoses assigned would be "Possible control" and the samples of this brain would be flagged to highlight its limitation. Thus, "Possible controls" are clearly segregated from "Normal controls". Samples from brains categorized as "Possible controls" are used in rare instances, for example, when tissues are requested to test or titer antibodies, or to adjust reagents, or to check techniques in progress. Furthermore, the distinction between "Normal control" and "Possible control" is made to optimize the validity of the interpretations of data these brains yield.

A list of request diagnoses (RDx) is included in a pulldown menu on the electronic request form, which is accessible at our web site (http://www.newyorkbrainbank. org). An investigator seeking a tissue can select the diagnosis of interest among those listed on the pull-down menu. If the one sought is not itemized, the investigators can specify the diagnosis they are interested in by using a dedicated field.

The software identifies samples in storage with characteristics that best match the variables of the request, as a link exists between the "distributive diagnosis" and the "matching diagnosis". The samples with the best complement between the "request diagnosis" and the "distributive diagnosis" are automatically identified. Simultaneously, the coordinates of the site where the eligible samples are stored are provided, allowing their retrieval from the storage freezers within minutes.

As mentioned, we perform a detailed, neuropathologic evaluation to characterize the variables of samples made 
available for research. We then generate two standardized reports based on the results of both macroscopic and microscopic examinations of the brain prepared for research. One report is text-based for the patient's clinicians and chart. The second is electronic and figure-based to assess the extent of the severity of any detectable changes. The resulting data are used to identify electronically, for example, a block of cerebral cortex (BA9) of a woman with mild dementia, which contains less than three neuritic plaques per $100 \times$ microscopic power. The software will provide a list of the matching samples for BA9, including the freezer coordinates to locate these samples of interest. Once they have been disbursed, a code indicates who performed the disbursement and to whom, where, and when the samples were sent. In addition, upon retrieval of stored samples (currently up to 500/month), the software records the space made available for newly harvested samples, with a consequential $45 \%$ increased efficiency of the freezer cabinets.

\section{Conclusion}

This report focuses on how best to prepare, categorize, and bank brains for research. The strengths of these methods are manifold. To ensure that the samples provided for research are truly representative of the disease of interest, only professionals knowledgeable about neuroanatomy, neuropathology, and the protocol, perform the dissections of the brains donated for research. To assess the brain tissue reliably, especially when it is processed fresh, expertise in neuropathology is necessary. Clinicopathological discrepancies of unsuspected, additional findings were discovered in $12 \%$ of brains collected for research over a 22-month period [49]. A significant difference in detection of abnormalities in 100 brains was documented, depending on whether the doctor had had formal postgraduate training in pathology. Furthermore, the difference was greater when the evaluation was done with fresh than with formalin-fixed brains [31].

To meet the needs for research and to secure the diagnostic categorization, one half-brain is banked and the other is processed for the neuropathological evaluation. Thus, one half-brain is processed fresh as soon as possible after death. The yield of extensively harvested and freshly processed samples is maximized, which produces a pool of specimens that have been precisely categorized, anatomically and diagnostically, and are eligible for a wide range of investigations. The availability of many stored samples is essential for fulfilling tissue requests quickly and optimally. An efficient operation demands a rigorous system for storing the samples. Briefly, a set of variables characterizes the samples stored in hierarchical, compartmentalized freezer cabinets. The variables are used to swiftly sort candidate samples that best match even complex investigations. These variables are electronically recorded and coupled with a barcoded tracking system for each stored sample. The identification and location of samples eligible for a specific investigation, is achieved quickly.

The core of these methods resides in the standardization of preparation and categorization of brains for research. The time invested for harvesting and freezing topographically well-categorized samples upon reception of a fresh brain is worthwhile. Indeed, these electronically tracked samples can be immediately disbursed once categorized diagnostically and qualitatively. There is no need, for example, to warm up a banked half-brain that was frozen en bloc, or a frozen coronal slice, to carve out a block including the amygdaloid nucleus. That approach is cumbersome; its steps are labor intensive; the temperature fluctuations inevitably stress the tissue. Moreover, keeping a record of the parts disbursed is challenging (Table 1).

A thorough, neuropathological examination using the contralateral half-brain is an integral part of the teaching function of the institution, which is important in light of the current low rate of autopsies. Furthermore, the standardization of the protocol establishes a collection of histological slides systematically selected from specific areas. Additionally, a collection of uniformly selected formalin phosphate-fixed and paraffin-embedded blocks are made available as a source of epitopes or nucleic acids, with specific topographies, abnormalities, and pedigrees.

This improved protocol has been successfully applied while processing about 500 brains at the NYBB at Columbia University since 2001. Currently, the NYBB manages more than 150,000 electronically tracked frozen samples. Up to 6,000 samples a year are disbursed worldwide. Ninety percent of the samples are disbursed to investigators within five working days from the time of receipt of the request forms.

Acknowledgments This work was supported by grants from the National Institutes of Health and National Institute on Aging: P01AG07232, R37-AG15473, and P50-AG08702, the Hereditary Disease Foundation, and the Iseman Fund. The authors thank Professor Kurt Jellinger for his insightful comments. We are grateful to Lisle Merriman for her editorial support, and to Mkeba Cason, Etty Cortes, M.D., and Katerina Mancevska for their help. The New York Brain Bank (NYBB) is especially thankful to the numerous pathologists who referred case material, and to the families of the patients for providing brain tissue for research.

\section{References}

1. Albrecht J, Yanagihara T (1979) Effect of anoxia and ischemia on ribonuclease activity in brain. J Neurochem 32:1131-1133 
2. Anderson AJ, Su JH, Cotman CW (1996) DNA damage and apoptosis in Alzheimer's disease: colocalization with c-Jun immunoreactivity, relationship to brain area, and effect of postmortem delay. J Neurosci 16:1710-1719

3. Aquilonius SM, Eckernäs SA, Sundwall A (1975) Regional distribution of choline actyltransferase in the human brain: changes in Huntington's chorea. J Neurol Neurosurg Psychiatry 38:669677

4. Bahn S, Augood SJ, Ryan M, Standaert DG, Starkey M, Emson PC (2001) Gene expression profiling in the post-mortem human brain — no cause for dismay. J Chem Neuroanat 22:79-94

5. Barton AJ, Pearson RC, Najlerahim A, Harrison PJ (1993) Pre- and postmortem influences on brain RNA. J Neurochem 61:1-11

6. Barton AJL, Hardy JA (1987) Stability of brain RNA post mortem: effect of Alzheimer's disease. Biochem Soc Trans 15:558559

7. Bird ED, Vonsattel JP (1993) The development of a brain bank. J Neural Transm Suppl 39:17-23

8. Braak H, Alafuzoff I, Arzberger T, Kretzschmar H, Del Tredici K (2006) Staging of Alzheimer disease-associated neurofibrillary pathology using paraffin sections and immunocytochemistry. Acta Neuropathol 112:389-404. doi:10.1007/s00401-006-0127-z

9. Braak H, Braak E, Bohl J (1993) Staging of Alzheimer related cortical destruction. Eur Neurol 33:403-408

10. Braak H, Del Tredici K, Rüb U, de Vos RAI, Steur ENH, Braak E (2003) Staging of brain pathology related to sporadic Parkinson's disease. Neurobiol Aging 24:197-211

11. Brodmann K (1908) Beiträge zur histologischen Lokalisation der Grosshirnrinde. VI. Mitteilung. Die Cortexgliederung des Menschen. J f Psychologie u Neurol 10:231-246

12. Burke WJ, O'Malley KL, Chung HD, Harmon SK, Miller JP, Berg L (1991) Effect of pre- and postmortem variables on specific mRNA levels in human brain. Mol Brain Res 11:37-41

13. Culliton BJ (1989) Wanted: normal brains. Science 243:889

14. Dodd PR, Hambley JW, Cowburn RF, Hardy JA (1988) A comparison of methodologies for the study of functional transmitter neurochemistry in human brain. J Neurochem 50:13331345

15. Dournaud P, Cervera-Pierot P, Hirsch E, Javoy-Agid F, Kordon C, Agid Y, Epelbaum J (1994) Somatostatin messenger RNAcontaining neurons in Alzheimer's disease: an in situ hybridization study in hippocampus, parahippocampal cortex and frontal cortex. Neuroscience 61:755-764

16. Duyckaerts C, Sazdovitch V, Seilhean D, Delaère P, Hauw JJ (1993) A brain bank in a neuropathology laboratory (with some emphasis on diagnostic criteria). J Neural Transm Suppl 39:107118

17. Ferrer I, Santpere G, Arzberger T, Bell J, Blanco R, Boluda S, Budka H, Carmona M, Giaccone G, Krebs B, Limido L, Parchi P, Puig B, Strammiello R, Ströbel T, Kretzschmar H (2007) Brain protein preservation largely depends on the postmortem storage temperature: implications for study of proteins in human neurologic diseases and management of brain banks: a BrainNet Europe study. J Neuropathol Exp Neurol 66:35-46

18. Grinberg LT, de Lucena Ferretti RE, Farfel JM, Leite R, Pasqualucci CA, Rosemberg S, Nitrini R, Saldiva PHN, Filho WJ (2007) Brain bank of the Brazilian aging brain study group-a milestone reached and more than 1,600 collected brains. Cell Tissue Banking 8:151-162. doi:10.1007/s10561-006-9022-Z

19. Hansen LA, Masliah E, Galasko D, Terry RD (1993) Plaque-only Alzheimer disease is usually the Lewy body variant, and vice versa. J Neuropathol Exp Neurol 52:648-654

20. Hansen LA, Samuel W (1997) Criteria for Alzheimer's disease and the nosology of dementia with Lewy bodies. Neurology 48:126-132
21. Harrison PJ (1999) The neuropathology of schizophrenia. A critical review of the data and their interpretation. Brain 122:593624

22. Harrison PJ, Procter AW, Barton AJL, Lowe SL, Najlerahim A, Bertolucci PHF, Bowen DM, Pearson RCA (1991) Terminal coma affects messenger RNA detection in post mortem human temporal cortex. Mol Brain Res 9:161-164

23. Harrison PJ, Procter AW, Exworthy T, Roberts GW, Najlerahim A, Barton AJ, Pearson RC (1993) Heat shock protein (hsx70) mRNA expression in human brain: effects of neurodegenerative disease and agonal state. Neuropathol Appl Neurobiol 19:10-21

24. Hasson J, Schneiderman H (1995) Autopsy training programs. To right a wrong. Arch Pathol Lab Med 119:289-291

25. Hebert LE, Scherr PA, Bienias JL, Bennet DA, Evans DA (2003) Alzheimer disease in the US population. Arch Neurol 60:11191122

26. Heckers S, Konradi C (2002) Hippocampal neurons in schizophrenia. J Neural Transm 109:891-905

27. Hulette CM (2003) Brain banking in the United States. J Neuropathol Exp Neurol 62:715-722

28. Hulette CM, Welsh-Bohmer KA, Crain B, Szymanski MH, Sinclaire NO, Roses AD (1997) Rapid brain autopsy. The Joseph and Kathleen Bryan Alzheimer's Disease Research Center experience. Arch Pathol Lab Med 121:615-618

29. Ikuta F, Hirano A, Zimmerman AW (1963) An experimental study of post-mortem alterations in the granular layer of the cerebellar cortex. J Neuropathol Exp Neurol 22:581-593

30. Jellinger KA, Lantos PL, Mehraein P (1993) Pathological assessment of movement disorders: requirements for documentation in brain banks. J Neural Transm Suppl 39:173-184

31. Katelaris A, Kencian J, Duflou J, Hilton JMN (1994) Brain at necropsy: to fix or not to fix? J Clin Pathol 47:718-720

32. Kingsbury AE, Foster OJF, Nisbet AP, Cairns N, Bray L, Eve DJ, Lees AJ, Marsden CD (1995) Tissue $\mathrm{pH}$ as an indicator of mRNA preservation in human post-mortem brain. Mol Brain Res 28:311-318

33. Mirra SS, Heyman A, McKeel D, Sumi SM, Crain BJ, Brownlee LM, Vogel FS, Hughes JP, van Belle G, Berg L (1991) The Consortium to Establish a Registry for Alzheimer's Disease (CERAD). Part II. Standardization of the neuropathologic assessment of Alzheimer's disease. Neurology 41:479-486

34. Monfort JC, Javoy-Agid F, Hauw JJ, Dubois B, Agid Y (1985) Brain glutamate decarboxylase in Parkinson's disease with particular reference to a premortem severity index. Brain 108:301313

35. Palmer AM, Lowe SL, Francis PT, Bowen DM (1988) Are postmortem biochemical studies of human brain worthwhile? Biochem Soc Trans 16:472-475

36. Perrett CW, Whatley SA, Ferrier IN, Marchbanks RM (1992) Changes in relative levels of specific brain mRNA species associated with schizophrenia and depression. Mol Brain Res 12:163-171

37. Perry EK, Perry RH (1983) Human brain neurochemistry-some postmortem problems. Life Sci 33:1733-1743

38. Petito CK, Roberts B (1995) Effect of postmortem interval on in situ end-labeling of DNA oligonucleosomes. J Neuropathol Exp Neurol 54:761-765

39. Price RA, McCormick WF (1981) The declining autopsy rate and its significance for neuropathology: two viewpoints. J Neuropathol Exp Neurol 40:489-492

40. Ravid R, van Zwieten EJ, Swaab DF (1992) Brain banking and the human hypothalamus-factors to match for, pitfalls and potentials. Prog Brain Res 93:83-95

41. Reynolds GP, Pearson SJ (1993) Neurochemical-clinical correlates in Huntington's disease-applications of brain banking techniques. J Neural Transm Suppl 39:207-214 
42. Ross BM, Knowler JT, McCulloch J (1992) On the stability of messenger RNA and ribosomal RNA in the brains of control human subjects and patients with Alzheimer's disease. J Neurochem 58:1810-1819

43. Schmitt A, Bauer M, Heinsen H, Feiden W et al (2007) How a neuropsychiatric brain bank should be run: a consensus paper of Brainnet Europe II. J Neural Transm 114:527-537. doi: 10.1007/s00702-006-0601-8

44. Spokes EG, Garrett NJ, Iversen LL (1979) Differential effects of agonal status on measurements of GABA and glutamate decarboxylase in human post-mortem brain tissue from control and Huntington's chorea subjects. J Neurochem 33:773-778

45. Stopa EG, Bird ED (1989) Brain donation. New Engl J Med $320: 62-63$

46. The National Institute on Aging and Reagan Institute Working Group on Diagnostic Criteria for the Neuropathological Assessment of Alzheimer's Disease (1997) Consensus recommendations for the postmortem diagnosis of Alzheimer's disease. Neurobiol Aging 18(Suppl 4):S1-S2

47. Tourtellotte WW, Rosario IP, Conrad A, Syndulko E (1993) Human neuro-specimen banking 1961-1992. J Neural Transm Suppl 39:5-15
48. Troncoso JC, Sukhov RR, Kawas CH, Koliatsos VE (1996) In situ labeling of dying cortical neurons in normal aging and in Alzheimer's disease: correlations with senile plaques and disease progression. J Neuropathol Exp Neurol 55:1134-1142

49. Vonsattel J-PG, Aizawa H, Ge P, DiFiglia M, McKee AC, MacDonald M, Gusella JF, Landwehrmeyer B, Bird ED, Richardson EP Jr, Hedley-Whyte ET (1995) An improved approach to prepare human brains for research. J Neuropathol Exp Neurol $54: 42-56$

50. Walker E, McNicol AM (1992) In situ hybridization demonstrates the stability of mRNA in post-mortem rat tissues. J Pathol 168:67-73

51. Witelson SF, McCulloch PB (1991) Premortem and postmortem measurement to study structure with function: a human brain collection. Schizophr Bull 17:584-591

52. Zarbo RJ, Baker PB, Howanitz PJ (1996) Quality assurance of autopsy permit form information, timeliness of performance, and issuance of preliminary report. A College of American Pathologists Q-Probes study of 5,434 autopsies from 452 institutions. Arch Pathol Lab Med 120:346-352 